\title{
Dynamics of cooling viscoplastic domes
}

\author{
By N. J. BALMFORTH ${ }^{1}$, R. V. CRASTER AND R. SASSI \\ ${ }^{1}$ Department of Applied Mathematics and Statistics, University of California, Santa Cruz, \\ CA 95064, USA \\ ${ }^{2}$ Department of Mathematics, Imperial College of Science, Technology and Medicine, \\ London SW7 2BZ, UK
}

(Received 30 October 2002 and in revised form 3 September 2003)

A variety of problems in engineering and geology involve spreading cooling nonNewtonian fluids. If the fluid is relatively shallow and spreads slowly, lubrication-style asymptotic approximations can be used to build reduced models for the spreading dynamics. The centrepiece of such models is a nonlinear diffusion equation for the local fluid thickness, and ideally this should become coupled to a correspondingly simple equation determining the local temperature field. However, when heat diffuses relatively slowly as the fluid flows, we cannot usefully reduce the temperature equation, and the asymptotic reduction couples the local thickness equation to an advection diffusion equation that crucially involves diffusion in the vertical. We present an efficient computational algorithm for numerically solving this more complicated type of lubrication model, and describe a suite of solutions that illustrate the dynamics captured by the model in the case of an expanding Bingham fluid with a temperaturedependent viscosity. Based on these solutions, we evaluate two simpler models that further approximate the temperature equation: a vertically isothermal theory, and a 'skin theory'. The latter is based on the integral-balance method of heat-transfer theory, and demands that the vertical structure of the temperature field has the form of an advancing boundary layer, or skin. The vertically isothermal model performs well when the thermal conductivity is relatively large. The skin theory reproduces the full dynamics qualitatively, if not quantitatively, for all thermal conductivities. The main errors in both models arise near the fluid edge, where the numerical solutions show that chilled fluid is overridden as the fluid expands, creating an underlying collar of cold material. Encouraged by the success of the skin model, we extend the theory by incorporating extensional stresses in the skin, which emerge when cooling induces an extreme rheological change in the material, such as an exponential rise in the viscosity. The model predicts that when skin stresses are sufficiently strong, the skin is brought to rest, whilst hotter fluid expands underneath.

\section{Introduction}

In many contexts in engineering and geology, the central problem surrounds how a cooling non-Newtonian fluid spreads on extrusion from a source, or as it slumps to rest when suddenly released. The applications range from lavas (Griffiths 2000), solid-state mantle plume heads (Bercovici \& Lin 1996) and glaciers (Hutter 1983) in geological settings, to molten films in chemical engineering (Braun et al. 1995) and coolants in the nuclear industry (Dinh, Konovalikhin \& Sehgal 2000). Related problems arise if the spreading liquids are heated or influenced by forces generated by changes in the concentration of a chemical either suspended or dissolved within the fluid (Reisfeld, Bankoff \& Davis 1991; Oron, Davis \& Bankoff 1997; Chomaz 2001). 
When the fluid is relatively shallow and spreads slowly, we can often take advantage of lubrication-style asymptotic approximations of the governing equations to build simpler mathematical models of the spreading dynamics. The philosophy of the lubrication approximation centres around the idea that the slenderness of the flow can be used to filter out the dependence on the depth coordinate: asymptotically, we solve explicitly for the fluid's vertical structure, thereby eliminating that spatial variable. For isothermal flows, the result of the asymptotic machinations is a simplified model which typically takes the form of a nonlinear diffusion equation for the local fluid thickness. When the fluid is also non-isothermal, we would ideally like to generalize the theory by performing a similar reduction of the temperature (or concentration) equation. The thickness equation then becomes coupled to a comparably simplified equation describing the local temperature field, as described by Balmforth \& Craster (2000, hereinafter referred to as paper 1) and Bercovici \& Lin (1996) in two geological problems. A review of this approach in other contexts is given by Oron et al. (1997).

Unfortunately, the mathematical modelling becomes significantly more complicated if the temperature diffuses relatively slowly. In this instance, the dimensional reduction of standard lubrication theory fails because heat does not diffuse sufficiently quickly to relax the temperature field to some local profile with a specified vertical structure. Instead, we must keep track of detailed vertical structure in the diffusing field, which amounts to solving the full advected heat equation. In other words, on proceeding through the asymptotic theory, some simplification of the governing equations results, but we cannot eliminate the depth coordinate from the final evolution equations that emerge.

The purpose of the current article is to explore lubrication theory for a spreading fluid with a slowly diffusing temperature field. This is precisely the physical state encountered in several geological settings because the thermal conductivity of both ice and lava is relatively small. A number of chemical engineering problems also have this feature, in either thermocapillarity (Ehrhard \& Davis 1991; Oron \& Rosenau 1994) or where the diffusing substance is a chemical species (Jensen \& Grotberg 1993; Hosoi \& Bush 2001; Chomaz 2001). At present, much of the existing literature assumes either rapid diffusion across the layer, as in the conventional lubrication approach, or that the chemical is an insoluble surfactant with no concentration whatsoever below the surface. Either way, the number of space dimensions can be reduced. However, the intermediate regime of moderate solubility and slow diffusion is not well studied.

The present theory is aimed largely at the modelling of evolving lava domes or their experimental analogues (Griffiths 2000). With this application in mind, and to keep the analysis fairly simple, we ignore many of the complicating physical effects that arise in real fluids, such as surface tension, capillarity and solidification. Even so, certain non-Newtonian effects are essential to this application, namely the existence of a yield stress and strongly temperature-dependent material parameters (silicic lavas have considerable yield stresses and the viscosity varies by several orders of magnitude on cooling, see paper 1). Hence, we must consider a non-isothermal viscoplastic fluid, which requires some choice for a rheological model. We adopt the Bingham model, and allow the viscosity to depend on temperature, $T$ :

$$
\left.\begin{array}{ll}
\tau_{i j}=\left[\eta(T)+\tau_{p} / \dot{\gamma}\right] \dot{\gamma}_{i j} & \text { for } \tau \geqslant \tau_{p}, \\
\dot{\gamma}_{i j}=0 & \text { otherwise, }
\end{array}\right\}
$$

where $\tau_{i j}$ denote the components of the deviatoric stresses, $\dot{\gamma}_{i j}=\partial u_{i} / \partial x_{j}+\partial u_{j} / \partial x_{i}$ represents the rate-of-strain tensor in Cartesian coordinates, $\tau_{p}$ and $\eta(T)$ are the yield stress and viscosity, $\tau=\sqrt{\tau_{i j} \tau_{i j} / 2}$ and $\dot{\gamma}=\sqrt{\dot{\gamma}_{i j} \dot{\gamma}_{i j} / 2}$. The yield stress, $\tau_{p}$, provides a 
cutoff below which the material forms plugs of rigid unyielded material. For practical purposes, we assume that $\tau_{p}$ is independent of temperature, and adopt an exponential dependence for $\eta(T)$. In our conclusions, we remark further on cases in which the yield stress also depends on $T$.

We describe lubrication theory for this fluid in $\S 2$, and compare the model with some earlier theories. We solve the reduced model equations using a computational scheme specially designed and optimized for the purpose. Since the problem is prototypical of many others in related areas, a full description of the scheme is given in Appendix A. The numerical solutions themselves are described in $\S 3$, where we also draw some conclusions regarding the dynamics captured by the model.

Although the computations are straightforward, they are intensive. Moreover, the reduced model is cumbersome because it contains the full heat equation. This motivates us to consider two further, simpler models that more crudely approximate the heat equation: the vertically isothermal theory of paper 1, and a 'skin theory'. The first theory cannot be expected to work in the limit of low thermal conductivity, which is the essential motivation behind our full computations, and we observe its limitations later all too readily. The second theory is based on the integral-balance method of heat-transfer theory (Özişik 1968), in which the vertical structure of the temperature field is represented by a known function of depth that approximates the expected solution. In particular, guided by the numerical solutions, we use an integral-balance ansatz with the form of an advancing thermal boundary layer, or skin. The skin depth and the temperatures at the base and surface are parameters of the ansatz, but the boundary conditions force a certain relationship amongst them, allowing us to rebuild the entire temperature field from only the surface temperature. An analysis of the depth-integrated heat equation then provides an evolution equation for this variable, and completes the reduced model. The derivation of the skin theory is presented in $\S 4$, together with some typical results and a comparison of all three theories. The use of the integral-balance method in lubrication theory is not new several studies in chemical engineering have used the approximation (Reisfeld et al. 1991; Braun et al. 1995; see also Neri 1998). However, no previous studies solve the full thin-layer model to verify that the integral-balance approximation is a good one, and part of the motivation for the current work is to assess its performance.

In comparison to the full numerics, we find that the skin theory reproduces the full dynamics qualitatively, if not quantitatively, for most thermal conductivities. Encouraged by this success, in $\S 5$ we extend the theory by incorporating extensional (or longitudinal) stresses. Such stresses are normally ignored in lubrication theory because they are usually dominated by vertical shear stresses (except in free films, Erneux \& Davis 1993, and viscous threads, Papageorgiou 1995). However, when cooling induces an extreme rheological change in the material, such as an exponential rise in the viscosity, the extensional stresses can become significant at the surface. Such extreme viscosity variations are expected in the geological applications of our theory, and so it is important to assess their effect. Extensional stresses are incorporated straightforwardly in the skin model; $\S 5$ describes the generalization and explores how the spreading dynamics is modified.

\section{Lubrication theory}

\subsection{Formulation}

We begin from the governing fluid equations for an axisymmetric cooling dome spreading over a horizontal plane, expressed in polar coordinates, $(r, \theta, z)$, and in a 
suitable dimensionless form. The non-dimensionalization is similar to that described in paper 1; we introduce the lengthscale $L$ and speed $U$ to remove dimensions from the radial distance, $r$, and velocity, $u$, and a depth scale $H$ and speed $H U / L$ for the vertical counterparts, $z$ and $w$. Pressure, $p$, is scaled with units of $\rho g H$, where $\rho$ is density and $g$ is gravity, and the deviatoric stresses, $\tau_{i j}$, and $\dot{\gamma}_{i j}$ with units $\rho g H^{2} / L$ and $U / H$, respectively. We further adopt $T=T_{a}+\left(T_{e}-T_{a}\right) \Theta$, where $T_{a}$ and $T_{e}$ are the ambient and eruption temperatures. In the slow-flow (zero-Reynolds-number) approximation, the dimensionless equations are:

$$
\begin{gathered}
\left.\begin{array}{c}
0=-p_{r}+\epsilon \partial_{r} \tau_{r r}+\partial_{z} \tau_{r z}+\epsilon r^{-1}\left(\tau_{r r}-\tau_{\theta \theta}\right), \\
0=-p_{z}-1+\epsilon^{2} \partial_{r} \tau_{r z}+\epsilon \partial_{z} \tau_{z z}+\epsilon^{2} r^{-1} \tau_{r z},
\end{array}\right\} \\
\frac{1}{r}(r u)_{r}+w_{z}=0 \\
\Theta_{t}+u \Theta_{r}+w \Theta_{z}=\kappa\left[\Theta_{z z}+\frac{\epsilon^{2}}{r}\left(r \Theta_{r}\right)_{r}\right],
\end{gathered}
$$

and

$$
\left.\begin{array}{l}
\tau_{i j}=[A(\Theta)+B / \dot{\gamma}] \dot{\gamma}_{i j} \text { for } \tau \geqslant B, \\
\dot{\gamma}_{i j}=0 \text { for } \tau<B,
\end{array}\right\}
$$

where $A(\Theta) \equiv \eta / \eta_{e}, \eta_{e}$ is a reference value determined by the eruption temperature, $T_{e}$ (so $A(1)=1$ ), and except for the stress components, the subscripts indicate partial derivatives. The equations contain a number of dimensionless groups: most important is the aspect ratio, $\epsilon=H / L$, which is assumed small and used to organize the asymptotic expansion. The remaining two parameters are the inverse Péclet number, $\kappa$, and Bingham number, $B$, defined by

$$
\kappa=\frac{L \mathscr{K}}{\rho c_{p} H^{2} U}, \quad B=\frac{\tau_{p} H}{\eta_{e} U},
$$

where $c_{p}$ and $\mathscr{K}$ are the specific heat and thermal conductivity. Note that we have ignored frictional heating on the grounds that it is typically small for lava (paper 1) and any other source terms such as latent heat, because we omit solidification for simplicity (both are easily incorporated, see Balmforth, Burbidge \& Craster 2001). Also, as in standard lubrication theory, we have demanded that the horizontal pressure gradient balance the opposing viscous stresses, which requires $U=\rho g H^{3} / \eta_{e} L$.

At the fluid base, we take the fluid to be motionless and insulated, except over a vent of radius $r_{*}$ in which material at the eruption temperature is introduced with a given vertical velocity. Hence, on $z=0, u=w=\Theta_{z}=0$ for $r>r_{*}$, whereas $u=0$, $w=\Omega(r)$ and $\Theta=1$ for $r \leqslant r_{*}$, where $\Omega(r)$ specifes the incoming velocity profile. At the surface, the fluid is considered stress free and loses heat according to a prescribed cooling law:

$$
\left.\begin{array}{l}
\tau_{r z}-h_{r}\left(\epsilon \tau_{r r}-p\right)=0 \\
\epsilon \tau_{z z}-p-\epsilon^{2} h_{r} \tau_{r z}=0
\end{array}\right\} \quad \text { on } z=h(r, t)
$$

and

$$
\kappa\left[\Theta_{z}\right]_{z=h}=-\mu \alpha\left(\Theta_{s}\right),
$$

where $\mu$ is a dimensionless cooling parameter and $\alpha\left(\Theta_{s}\right)$ is a given, order one, function of the surface temperature, $\Theta_{s}(r, t)=\Theta(r, h, t)$. For example, with Newton's law, $\alpha\left(\Theta_{s}\right)=\Theta_{s}$. A more detailed discussion of the thermal boundary condition is 
given in paper 1 and by Neri (1998). There is also the kinematic condition,

$$
h_{t}+u(r, h, t) h_{r}=w(r, h, t) .
$$

\subsection{Shallow-lava model}

We now discard higher-order terms in $\epsilon$ in (2.1) to find:

$$
p_{r}=\partial_{z} \tau_{r z}, \quad p_{z}=-1 .
$$

Thus, $p=P_{s}+h-z$ and $\tau_{r z}=\tau_{s}-\left(P_{s r}+h_{r}\right)(h-z)$, where $P_{s}(r, t)$ and $\tau_{s}(r, t)$ denote the surface pressure and shear stress. Application of the surface boundary conditions indicates that $P_{s}=\tau_{s}=0$. Hence, $p=h-z$ and $\tau_{r z}=-h_{r}(h-z)$.

Also, from the constitutive law,

$$
\tau_{r z}=\left[A(\Theta)+\frac{B}{\left|u_{z}\right|}\right] u_{z},
$$

if $\tau=\left|\tau_{r z}\right|>B$, and $u_{z}=0$ otherwise. Thence,

$$
u_{z}= \begin{cases}-A^{-1} h_{r}(Y-z) & \text { for } z \leqslant Y(r, t), \\ 0 & \text { for } z>Y(r, t),\end{cases}
$$

where the surface, $z=Y(r, t)=\operatorname{Max}\left(h-B /\left|h_{r}\right|, 0\right)$, separates a lower layer of yielding shearing material from an upper region occupied by fluid which, at leading order, appears to flow like a rigid plug. In fact, analysis of higher-order terms (Balmforth \& Craster 1999) reveals that the upper region is actually weakly yielding, which resolves the apparent contradiction that an axisymmetrical expansion cannot be rigid. Consequently, we refer to the upper region as a pseudo-plug, and $z=Y(r, t)$ as a fake yield surface.

The velocity components now follow from quadrature, as does the depth-integrated radial velocity:

$$
\mathscr{U}(r, t)=\int_{0}^{h} u(r, z, t) \mathrm{d} z=-h_{r} \int_{0}^{Y} \frac{(Y-z)(h-z)}{A(\Theta)} \mathrm{d} z .
$$

This final quantity is required on combining the kinematic surface boundary condition with the integral of the continuity equation:

$$
h_{t}+\frac{1}{r} \frac{\partial}{\partial r}(r \mathscr{U})=\Omega(r, t) .
$$

Through the integral in (2.12), this equation for $h$ is coupled to the advected heat equation,

$$
\Theta_{t}+u \Theta_{r}+w \Theta_{z}=\kappa \Theta_{z z},
$$

which must be solved subject to the flux condition (2.7) and the thermal boundary conditions on the base.

We must also impose boundary conditions in radius. Symmetry demands that $h_{r}=\Theta_{r}=0$ at $r=0$. At the edge of the dome, $r=R(t)$, we should, in principle, insist that $h \rightarrow 0$, but the surface gradient can then become singular there (see Balmforth et al. 2000). We avoid this complication by placing a lower limit on the thickness of the fluid and imposing the boundary condition, $h=h_{w}$ for $r \geqslant R$, where $h_{w}$ is typically taken to be $10^{-4}$. We solve the equations on a computational domain that expands in time; an equation of motion for the edge of the domain can be found from the $h$-equation and solved in conjunction with the two partial differential equations. Further details are given in Appendix A. 
Dominant physics

(i) Bulk viscosity

(ii) Bulk yield stress

(iii) Bulk cold viscosity

(iv) Edge shear stress

(v) Extensional stress
$R(t)$

$t^{1 / 2}$

$B^{-1 / 5} t^{2 / 5}$

$A_{0}^{-1 / 8} t^{1 / 2}$

$t^{4 / 7}\left(\delta A_{0}\right)^{-1 / 7} \rightarrow\left(c A_{0}\right)^{-1 / 7} t^{1 / 2}$

$\left(A_{0} \delta\right)^{-1 / 4} t^{3 / 4} \rightarrow\left(c A_{0}\right)^{-1 / 4} t^{5 / 8}$ $h_{0}(t)$

$t^{0}$

$B^{2 / 5} t^{1 / 5}$

$A_{0}^{1 / 2} t^{0}$

$\left(\delta A_{0} / t^{1 / 2}\right)^{2 / 7} \rightarrow\left(c A_{0}\right)^{2 / 7}$

$\left(A_{0} \delta / t\right)^{1 / 2} \rightarrow\left(c A_{0}\right)^{1 / 2} t^{-1 / 4}$

TABLE 1. Various scalings that emerge by balancing the gravitational driving force with retarding forces acting over either the bulk of the fluid, over a surface layer of depth $\delta$, or over an edge layer of thickness $\delta$ (cf. Griffiths \& Fink 1993). In the latter cases, we then take $\delta=c t^{1 / 2}$; arguments presented later suggest the estimate $c=2 \sqrt{3 \kappa}$.

At this stage, it is useful to observe that there is still sufficient freedom in the dimensional scalings to eliminate one of the dimensionless parameters. The lubrication balance, $U=\rho g H^{3} / \eta L$, and the dimensional volumetric flow rate, $Q=H L U$, relate $U$ and $H$ to $L$, but $L$ is currently undetermined. We select $L$ so that the fluid surface cools over an order unity timescale. Specifically, the surface cooling time is $O\left(\kappa / \mu^{2}\right)$, and so we demand that $\mu=\sqrt{\kappa}$. With this choice, the thermal boundary condition becomes $-\sqrt{\kappa}\left[\Theta_{z}\right]_{z=h}=\alpha\left(\Theta_{s}\right)$, and we are left with the parameters, $\kappa, B$ and $r_{*}$. We further fix the vent radius as $r_{*}=0.05$, on the grounds that it plays a minor role once the fluid spans a much larger domain.

\subsection{Previous results}

Before we go about solving the shallow-lava model numerically (which is the focus of the next section), we first relate the theory to some previously derived, simpler models. We loosely classify these as either dimensional scaling theories, isothermal models, or vertically isothermal theories. Because of their greater complication, full thin-layer models have received less attention in the past, with one notable exception: a relatively low thermal diffusivity is a characteristic feature of ice flow, and most models of glaciers and ice sheets build in a thin-layer heat equation (Hutter 1983). Despite this similarity, ice is not commonly assumed to possess a yield-stress (Glen's law, which is usually used to model ice, is equivalent to a power-law rheology) and typically flows in the form of roughly steady streams, fed from upstream (by either an ice reservoir or from snow accumulation) and dissipated downstream (by ablation or outflow into water with its attendant calving of icebergs). A more closely related thin-layer theory was presented by King, Riley \& Sansom (2000) to model spreading coolants in nuclear engineering, although they too ignored yield stresses and primarily considered fixed volumes.

\subsubsection{Dimensional scaling}

Griffiths \& Fink (1993) predict maximum dome height $h_{0}(t)$ and radius $R(t)$ for cooling extrusions using dimensional analysis. We summarize these results and place them into our current notation as follows. For a constant-flux extrusion, the radius and height are connected by $h_{0} R^{2} \sim t$. Moreover, radial pressure gradients, $p_{r}=h_{r} \sim h_{0} / R$, drive expansion and must be balanced by fluid stresses. On equating the volumeaveraged driving force, $\iint p_{r} r \mathrm{~d} r \mathrm{~d} z \sim R h_{0}^{2}$, with the volume average of the dominant resistive stress, we can then extract the temporal behaviour of the maximum dome height and radius. In table 1, we give the dimensional scaling results obtained in this 
way, assuming the dominant resistive stresses to be:

(i) Vertical shear stress acting over fluid interior at the eruption temperature $\iint \partial_{z} \tau_{r z} r \mathrm{~d} r \mathrm{~d} z \sim R^{3} / h_{0} t$ with $\tau_{r z}=u_{z} \sim R /\left(h_{0} t\right)$.

(ii) Yield stress acting over fluid interior $\iint \partial_{z} \tau_{r z} r \mathrm{~d} r \mathrm{~d} z \sim R^{2} B$ with $\tau_{r z} \approx B$.

(iii) Vertical shear stress acting over fluid interior at the ambient temperature $\iint \partial_{z} \tau_{r z} r \mathrm{~d} r \mathrm{~d} z \sim R^{3} A_{0} / h_{0} t$ with $\tau_{r z}=A_{0} u_{z}$, and $A_{0}=A(0)$ representing the enhancement of the viscosity due to cooling to the ambient temperature.

(iv) Cooling-enhanced shear stress at fluid edge $\iint \partial_{z} \tau_{r z} r \mathrm{~d} r \mathrm{~d} z \sim R^{2} \delta A_{0} /\left(t h_{0}\right)$ with $\tau_{r z}=A_{0} u_{z}$ over a depth $h_{0}$, but radial extent $\delta$.

(v) Cooling-enhanced surface extensional stress $\iint \partial_{r} \tau_{r r} r \mathrm{~d} r \mathrm{~d} z \sim R \delta A_{0} / t$ with $\tau_{r r} \sim A_{0} u_{r}$ over a depth $\delta$.

The first three balances provide the scalings expected for either Newtonian domes or yield-stress-dominated fluids (Huppert 1982; Blake 1990). Balance (iv) indicates how expansion might be controlled by a cooling-enhanced shear stress in a cooled layer near the dome's rim (Griffiths \& Fink 1993; see also Stasiuk, Jaupart \& Sparks 1993 and Lister \& Kerr 1994), which further requires the time variation of the layer thickness, $\delta$. Griffiths \& Fink assume $\delta$ to grow like a stationary thermal boundary layer. Along these lines, we offer later the estimate $\delta \sim 2 \sqrt{3 \kappa}$, which leads to the explicit temporal dependence also given in the table. The final case introduces cooling-enhanced extensional stresses, which are incorporated in a more complete way in our revision of the skin theory in $\S 5$; as it turns out, the scaling result (v) is misleading because extensional stresses arrest the expansion of the cooled surface layers, but not the hotter fluid beneath.

\subsubsection{Isothermal flow}

When cooling is unimportant, the heat equation can be ignored and $A(\Theta) \rightarrow 1$, leaving

$$
h_{t}=\frac{1}{6 r} \frac{\partial}{\partial r}\left[r Y^{2} h_{r}(3 h-Y)\right]+\Omega .
$$

This equation was explored in Balmforth et al. (2000). Various similarity solutions appropriate to the Newtonian or yield-stress-dominated limits reproduce the dimensional scaling results described above for flows controlled by bulk viscosity or yield stress.

\subsubsection{Vertically isothermal theories}

When $\kappa \gg 1$, diffusion rapidly smooths temperature variations in the vertical, leading to local thermal equilibrium at each radius. For the current problem, the smoothed temperature field is uniform in $z$ (paper 1), but alternative profiles can be established with different boundary conditions (Bercovici \& Lin 1996). We reduce the thin-layer model accordingly as follows. We integrate the heat equation over the fluid depth, and apply the thermal boundary conditions. We then make the approximate replacement, $\Theta(r, z, t) \rightarrow \Theta(r, t)$, and evaluate the mass flux using $\Theta$. This generates the model:

$$
h_{t}+\frac{1}{r}(r \mathscr{U})_{r}=\Omega, \quad \check{\Theta}_{t}+\frac{\mathscr{U}}{h} \check{\Theta}_{r}=-\frac{\mu}{h} \alpha(\check{\Theta})+\frac{\Omega}{h}(1-\check{\Theta}), \quad \mathscr{U}=\frac{h_{r} Y^{2}(3 h-Y)}{6 A(\check{\Theta})} .
$$

Note that, in contrast to paper 1 , we non-dimensionalize the viscosity with its value at the eruption temperature, rather than that at the ambient temperature, since the former plays a greater role in less diffusive domes. 

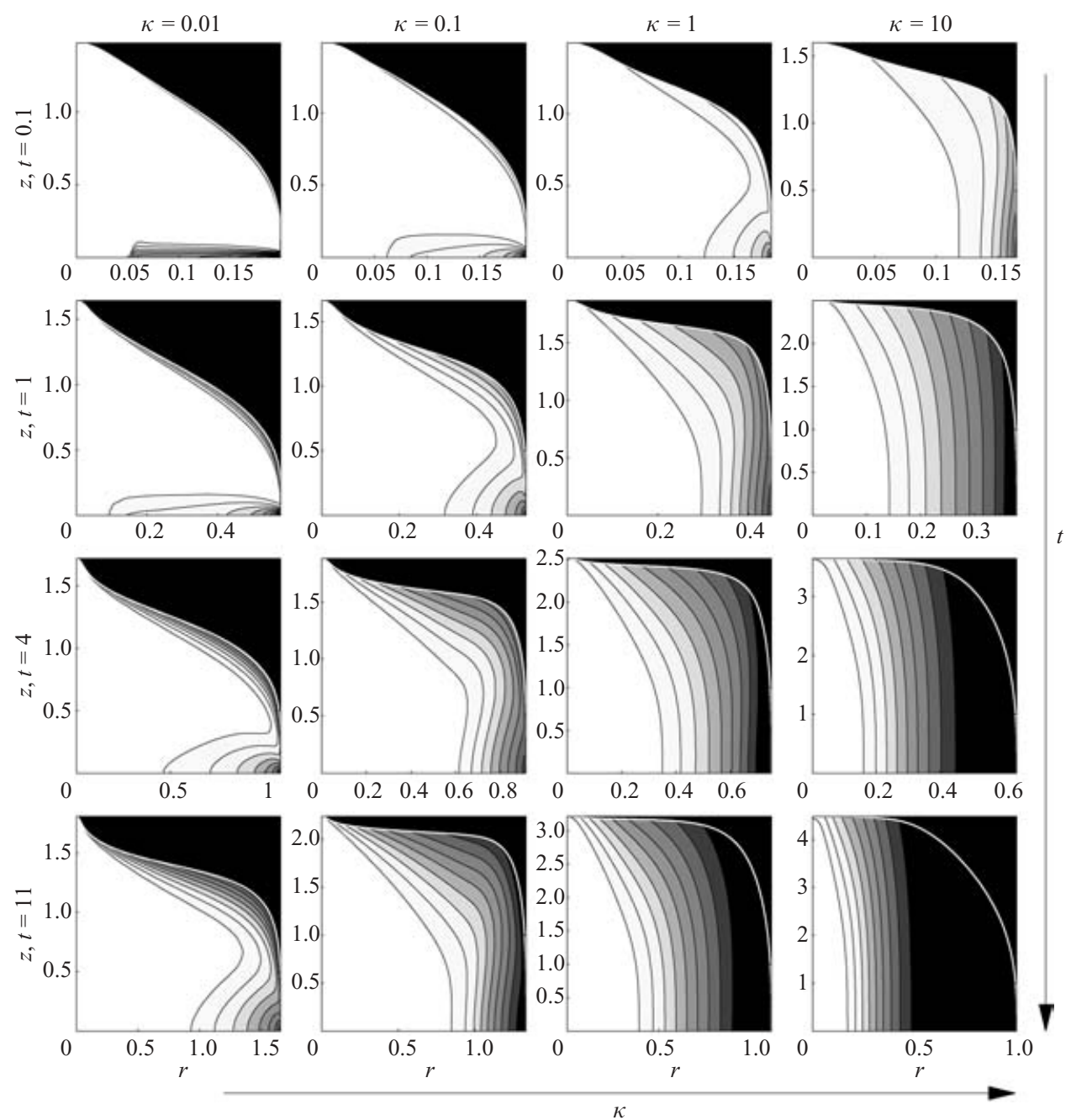

FIGURE 1. Snapshots of evolving domes: Four numerical computations with $\gamma=6$ and $B=0$. Shown are contours of constant temperature on the $(r, z)$-plane; contours are spaced by 0.1 , from 0 (black: cold) to 1 (white: hot). Each column represents a computation with different inverse Péclet number: $\kappa=0.01,0.1,1$ and 10. Each row represents a snapshot at a given time: $t=0.1,1,4$ and 11 .

\section{Computational results}

In figures $1-8$, we show a collage of results that illustrate the dynamics contained in the shallow lava model. For the purposes of illustration, we take the cooling law and viscosity function,

$$
\alpha\left(\Theta_{s}\right)=\Theta_{s}, \quad A(\Theta)=\exp \gamma(1-\Theta),
$$

where $\gamma$ is a parameter that determines the viscosity variation over the imposed temperature range, and the mass source to be given by the constant-in-time flux function,

$$
\Omega(r)=\frac{3}{\pi r_{*}^{2}}\left[\operatorname{Max}\left(1-\frac{r^{2}}{r_{*}^{2}}, 0\right)\right]^{2},
$$

(with $r_{*}=0.05$ ). The initial condition is $h(r, 0)=h_{w}=10^{-4}$ and $\Theta(x, z, 0)=1$. 
(a)
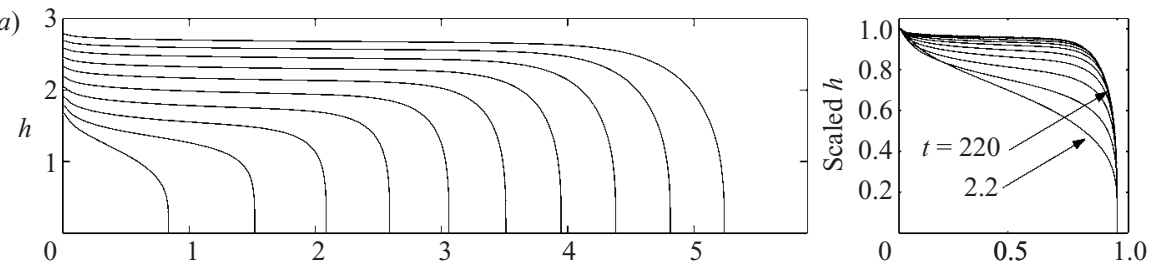

(b)
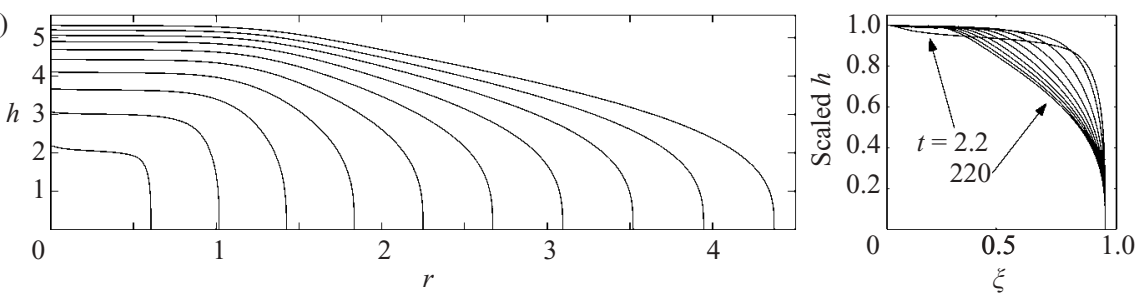

FiguRE 2. Snapshots of the dome thickness at the times $t=2.2 n^{2}, n=1,2, \ldots, 10$ for $(a)$ $\kappa=0.01$ and $(b) \kappa=1$, with $B=0$ and $\gamma=6$. The data are redrawn, scaling radius by the dome radius, $\xi=r / R$, and thickness by dome height, $h(r, t) / h(0, t)$, in the two smaller panels.
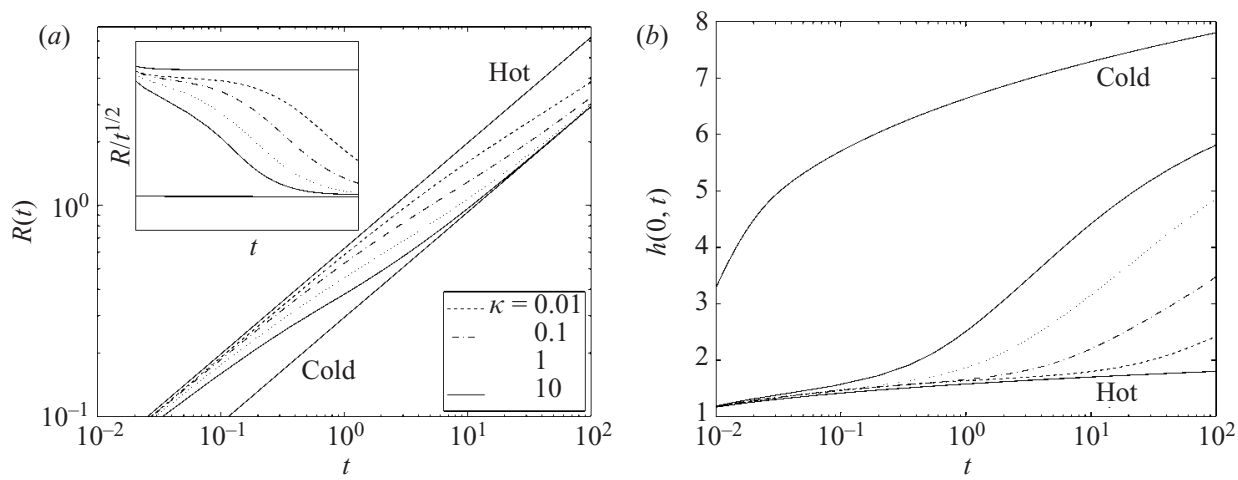

Figure 3. (a) Dome radius and (b) maximal height for the computations of figure 1 . $B=0, \gamma=6$. The solid curves marked hot and cold show isothermal extrusions for fluids at the eruption and ambient temperatures. The trends of the expected power laws for an isothermal Newtonian fluid are $h(0, t) \sim t^{0}$ and $R(t) \sim t^{1 / 2}$; the inset in $(a)$ shows $R(t) / t^{1 / 2}$ versus $t$.

Figures 1-4 show results for $B=0$ and $\gamma=6$ (giving a viscosity variation of about three orders of magnitude over the temperature range). Roughly speaking, all the extrusions begin with an outflow of hot material that expands at the eruption temperature $(\Theta=1)$. The material begins to cool at the surface, but initially the isotherms expand with the fluid. The expanding cooled rim of the dome dams up the hotter fluid behind the flow front, causing the edge of the dome to steepen with time (see the computation with $\kappa=0.01$ in figure 2). Subsequently, after a time of order $1 / \kappa$, the temperature field diffuses to an almost steady profile. Thereafter, the fluid expands like an isothermal dome at the ambient temperature $(\Theta=0)$. During the later stages, the rim no longer dams up the flow, and the dome sides slump to become less steep, as illustrated by the computation with $\kappa=1$ in figure 2 . Further details of the expansion are shown in figures $3-4$, which show the evolution of the dome radius and height and the relaxation of the temperature profile for some of the extrusions of figure 1 . 

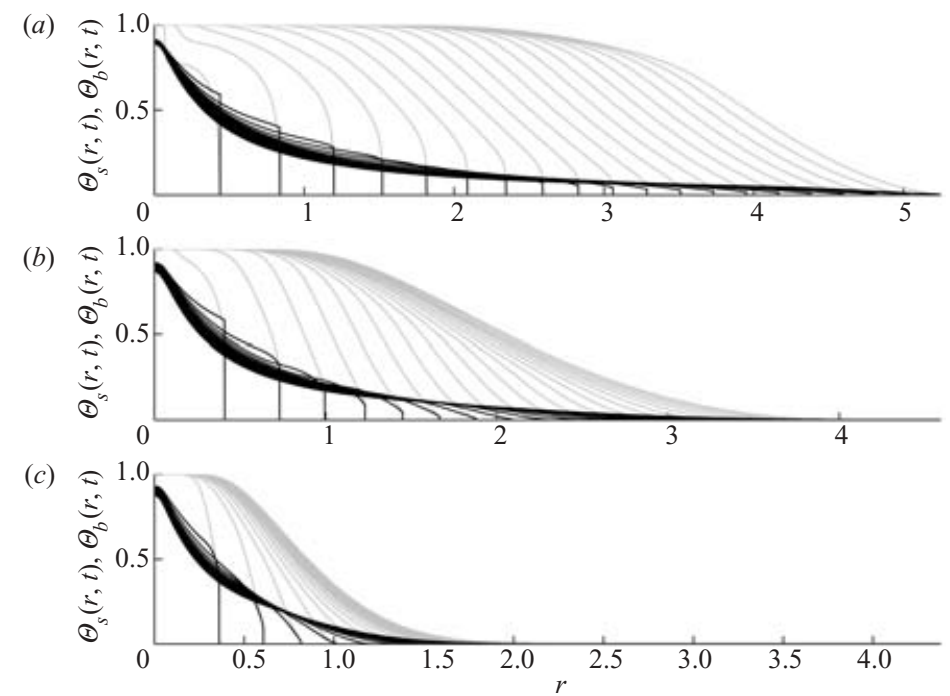

Figure 4. Snapshots of the surface and basal temperature profiles for three of the computations of figure 1 . Times are $t=0.55 n^{2}, n=1,2, \ldots, 20$. The darker lines show the surface temperature, whereas the lighter lines show the basal temperature. $(a) \kappa=0.01,(b) 0.1,(c) 1$.

When $\kappa$ is small (left-hand columns of figure 1), the bulk of the fluid expands for an extended period at the extrusion temperature. As the surface cools, the chilled fluid is swept along the surface and deposited at the dome edge. There, the cooled material becomes over-ridden by outflowing hot fluid, and creates an underlying layer of cold fluid. The overlying hot flow subsequently reheats the cold material deeper in the dome's interior, leaving an expanding, low-lying collar of cold material near the dome's rim, as observed by Stasiuk et al. (1993) in their experiments with cooling syrup.

For larger $\kappa$, diffusion acts more quickly, and the isotherms become nearly vertical as time proceeds (last column of figure 1). The overall dynamics is similar to that expected from the vertically isothermal theory (paper 1).

With finite yield stress, the general picture is much the same, see figures $5-7$. The first of these pictures displays snapshots at $t=11$ of various domes with different $B$ and $\kappa$. Also indicated is the fake yield surface, $z=Y(r, t)$, which becomes elevated in the cooler parts of the domes where the viscous stresses are higher (we observed this effect previously in the simpler model of paper 1). One difference with the $B=0$ results is that cooling in domes with large $B$ has a much smaller effect on the dynamics of the expanding flow (see figure 6). Even in cases with smaller $B$, the effect of surface cooling is more limited because the rheological variations are largely confined to the dome's pseudo-plug, where the yield stresses dominate the viscous shear stress. Moreover, the continued upward growth of the dome reduces the outward transport of cool material, and slows the build-up of cold fluid at the edge.

\section{Skin theory}

A main feature of the numerical solutions of the previous section is that, when $\kappa$ is small, cooling is concentrated at the upper surface of the fluid and a chilled boundary layer descends into the interior of the fluid as it expands. Actually, the solution for $\Theta$ can be surprisingly well represented by a piecewise continuous polynomial in $z$. 

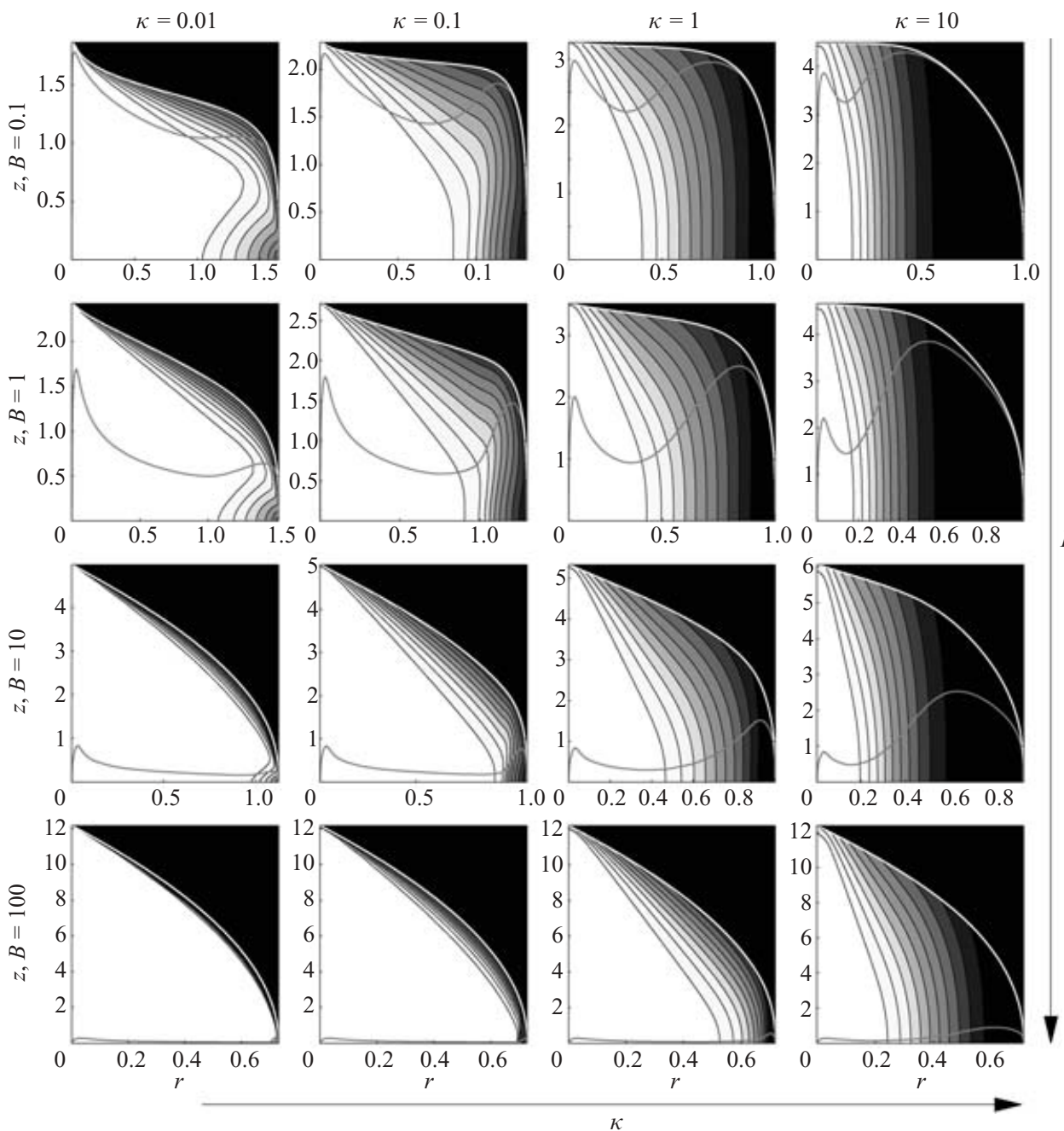

FIGURE 5. Snapshots at $t=11$ for four numerical computations with $\gamma=6$ and differing $\kappa$ and $B$. As in figure 1, each picture shows isotherms. Also shown by the lighter solid curve is the fake yield surface, $z=Y(r, t)$. Each column represents a computation with different inverse Péclet number: $\kappa=0.01,0.1,1$ and 10. Each row represents a different $B: 0.1,1,10$ and 100 .

This is illustrated in figure 8, which compares a snapshot of a numerical solution (for $B=\gamma=0$ ) with such a function. The approximation does not capture the low-lying collar of cold material, but works well everywhere else. This agreement motivates us to use the integral-balance method to build a simpler 'skin theory'. To whet the reader's appetite, we have also included the corresponding result from the skin theory in panel $(c)$ of figure 8 .

\subsection{Integral-balance solution}

We begin by integrating the heat equation (2.14), from a level, $z=h-\delta$, to the surface, $z=h$ :

$$
\left[\delta\left(\bar{\Theta}-\Theta_{b}\right)\right]_{t}+\frac{1}{r} \frac{\partial}{\partial r}\left[r \delta\left(\overline{u \Theta}-\bar{u} \Theta_{b}\right)\right]+\delta\left(\Theta_{b t}+\bar{u} \Theta_{b r}\right)=-\mu \alpha\left(\Theta_{s}\right)-\left.\kappa \Theta_{z}\right|_{z=h-\delta},
$$



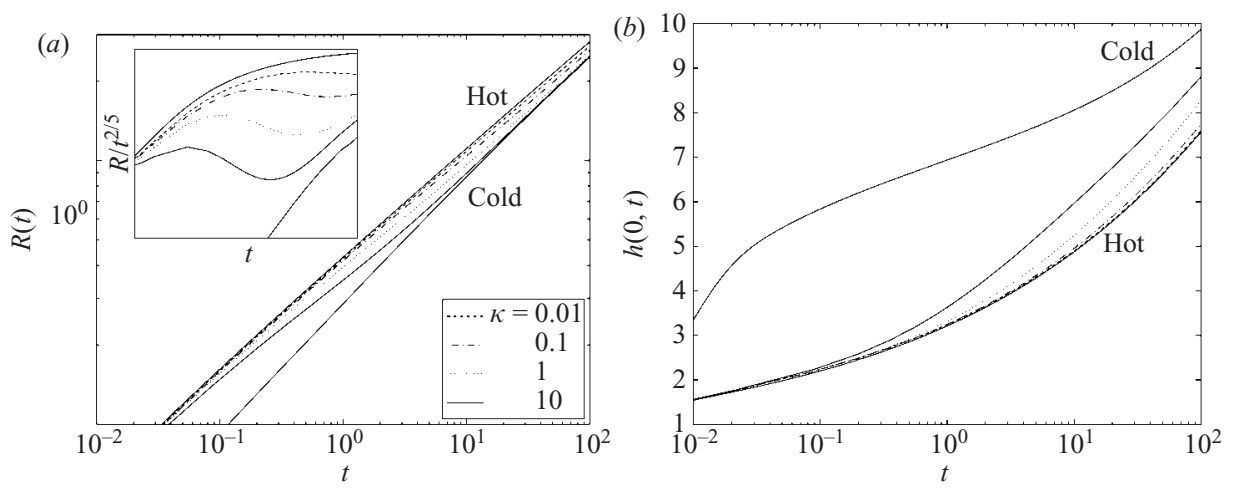

FIgURE 6. ( $a$ ) Dome radius and $(b)$ maximal height $v$ s. time for $B=10, \gamma=6$ and four values of $\kappa$. The curves marked hot and cold show isothermal extrusions for fluids at the eruption and ambient temperatures. For an isothermal yield-stress-dominated dome we expect $R(t) \sim t^{2 / 5}$; in $(a)$, the inset replots the data as $R / t^{2 / 5}$ versus $t$.

(a)

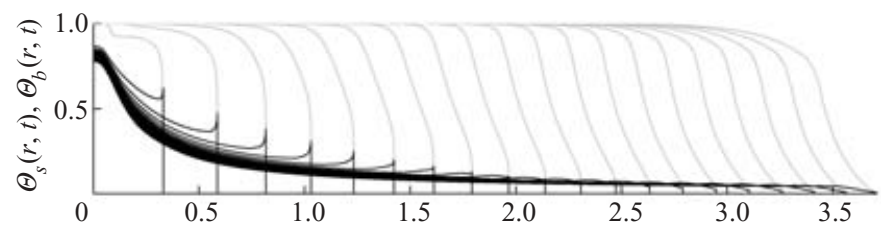

(b)

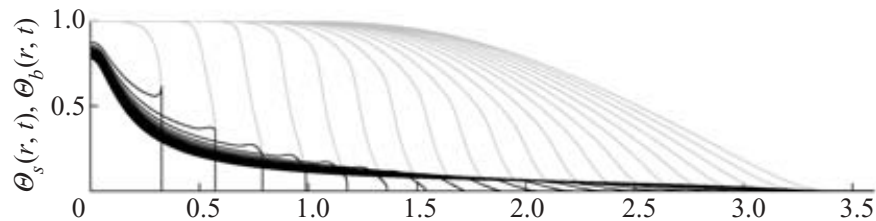

(c)

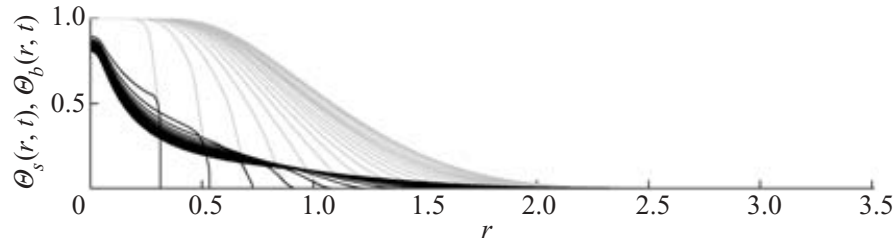

FIGURE 7. Snapshots of the surface and basal temperature profiles for three computations with $\gamma=6$ and $B=10$. Times are $t=0.55 n^{2}, n=1,2, \ldots, 20$. The darker lines show the surface temperature, whereas the lighter lines show the basal temperature. $(a) \kappa=0.01,(b) 0.1,(c) 1$.

where the bar indicates the vertical average,

$$
\bar{f}(z)=\delta^{-1} \int_{h-\delta}^{h} f(z) \mathrm{d} z,
$$

the subscripts $s$ and $b$ denote the values at the surface and base of the skin, and we have used the kinematic surface boundary condition and the continuity equation.

We now introduce the integral-balance ansatz for $\Theta(r, z, t)$, the piecewise continuous polynomial,

$$
\Theta= \begin{cases}\Theta_{b}-\left(\Theta_{b}-\Theta_{s}\right) \mathscr{P}(\zeta / \delta) & \text { for } \zeta \leqslant \delta \\ \Theta_{b} & \text { for } \zeta>\delta\end{cases}
$$




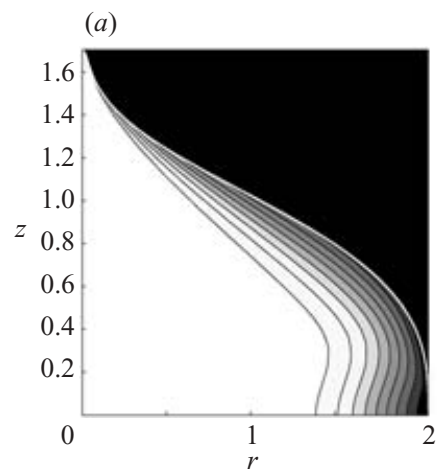

(b)

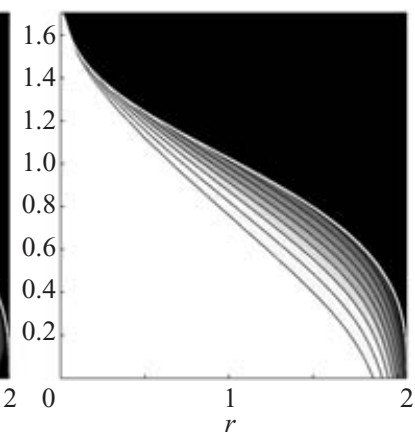

(c)

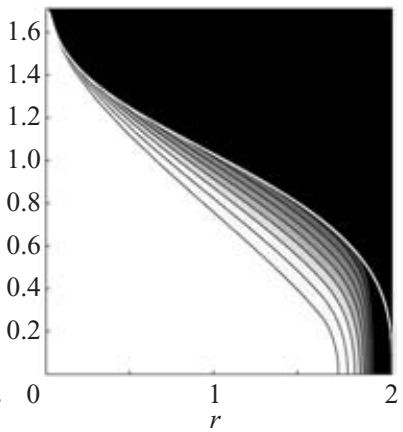

FIGURE 8. A comparison of the height and temperature fields at $t=11$ for a dome with $\kappa=0.01$, and $\gamma=B=0$. The panels show isotherms (spaced by 0.1 from 0 (black) to 1 (white)). Panel $(a)$ shows the full numerical solution, $(b)$ displays the profile computed from the surface temperature assuming the integral-balance ansatz for the temperature field $\left(\Theta=\Theta_{b}-\left(\Theta_{b}-\right.\right.$ $\left.\Theta_{s}\right)(1-(h-z) / \delta)^{2}$, where $\delta=\operatorname{Min}\left[2 \kappa^{1 / 2}\left(1-\Theta_{s}\right) / \Theta_{s}, h\right]$ and $\left.\Theta_{b}=\Theta_{s}(\delta+2 \sqrt{\kappa}) /(2 \sqrt{\kappa})\right)$, and $(c)$ shows the integral-balance solution computed using the skin theory of $\S 4$.

where $\zeta=h-z$ and $\mathscr{P}(x)$ is a known function with the properties, $\mathscr{P}(1)=\mathscr{P}^{\prime}(1)=0$ and $\mathscr{P}(0)=1$. A popular, convenient choice is $\mathscr{P}(x)=(1-x)^{m}$, for some $m>1$. For practical purposes, we adopt this form with $m=2$, as illustrated in figure 8. Certain choices of $\mathscr{P}(x)$ are presumably more accurate than others in given situations, but there is no obvious optimal selection. The integral-balance solution in (4.2) has the properties that the temperature field below the skin is uniform, and $\Theta_{z}=0$ at the skin's base. Thus, to be consistent with the initial condition, we take $\Theta_{b}=1$ if $h>\delta$. However, if the skin extends throughout the dome and touches the base, we take $\delta=h$ and allow $\Theta_{b}$ to decrease by cooling.

The integrated heat equation (2.14) can now be written in the form,

$$
[\delta(\bar{\Theta}-1)]_{t}+\frac{1}{r} \frac{\partial}{\partial r}[r \delta(\overline{u \Theta}-\bar{u})]=-\mu \alpha\left(\Theta_{s}\right),
$$

where we have used the fact that either $\Theta_{b}=1$, or $\delta=h$ and $h_{t}+r^{-1} \partial_{r}(r \delta \bar{u})=0$. Furthermore, with (4.2), we find the relations,

$$
\bar{\Theta}=\Theta_{b}-\left(\Theta_{b}-\Theta_{s}\right) \int_{0}^{1} \mathscr{P}(x) \mathrm{d} x=\Theta_{b}-c_{1}\left(\Theta_{b}-\Theta_{s}\right)
$$

and

$$
\Theta_{\zeta}(r, 0, t)=-\frac{1}{\delta}\left(\Theta_{b}-\Theta_{s}\right) \mathscr{P}^{\prime}(0)=\frac{c_{2}}{\delta}\left(\Theta_{b}-\Theta_{s}\right),
$$

for two constants $c_{1}$ and $c_{2}$ (equal to $1 / 3$ and 2 , respectively, for our polynomial selection). These lead to the expressions,

$$
\delta=\operatorname{Min}\left[\frac{\kappa c_{2}\left(\Theta_{b}-\Theta_{s}\right)}{\mu \alpha\left(\Theta_{s}\right)}, h\right], \quad \Theta_{b}=\Theta_{s}+\frac{\delta \mu \alpha\left(\Theta_{s}\right)}{\kappa c_{2}} .
$$

Hence, all variables determining the temperature field may be built from $\Theta_{s}$.

Finally, we define a convenient new variable,

$$
\mathscr{E}=\frac{\delta(1-\bar{\Theta})}{\mu c_{1} c_{2}}=\left[1-\Theta_{b}+c_{1}\left(\Theta_{b}-\Theta_{s}\right)\right] \frac{\delta}{\mu c_{1} c_{2}},
$$


to arrive at the reduced heat equation,

$$
\mathscr{E}_{t}+\frac{1}{r} \frac{\partial}{\partial r}(r \bar{u} \mathscr{E})-\frac{1}{r} \frac{\partial}{\partial r}\left[\frac{r \delta}{\mu c_{1} c_{2}}(\overline{u \Theta}-\bar{u} \bar{\Theta})\right]=\frac{1}{c_{1} c_{2}} \alpha\left(\Theta_{s}\right) .
$$

This equation contains advection by the vertically integrated radial velocity, with a correction accounting for the vertical structure of the temperature field, and surface cooling. There is no source term other than that arising from convergence of the flow, which deepens the skin layer. Thus, the skin always cools and is never replenished from beneath or from its edges.

\subsection{The velocity field}

The integral-balance solution divides the fluid into two regions: a superficial skin and an underlying isothermal interior. In the interior, $\Theta=A=1$. Thus, $p=P_{s}+h-z$ and $\tau_{r z}=\tau_{s}-\left(P_{s r}+h_{r}\right)(h-z)$, as in the shallow-lava model. When rheological changes are not severe, the stress components remain unchanged also within the skin, and we again find that $P_{s}=\tau_{s}=0$. The constitutive law then predicts that

$$
u_{z}=-h_{r} \operatorname{Max}(Y-z, 0), \quad Y=\operatorname{Max}\left(h-B /\left|h_{r}\right|, 0\right),
$$

and we again have a pseudo-plug riding over a lower shear layer. The velocity at the base of the skin can be written in the compact form, $u_{b}=-h_{r} Z(Y-Z / 2)$, where $Z=\operatorname{Min}(Y, h-\delta)$. Similarly, the depth-integrated radial velocity in the interior is

$$
\int_{0}^{h-\delta} u \mathrm{~d} z \equiv \mathscr{U}=(h-\delta) u_{b}-\frac{1}{6} h_{r} Z^{2}(2 Z-3 Y) .
$$

In the skin, the constitutive relation becomes

$$
A(\Theta) u_{z}=-h_{r} \operatorname{Max}(Y-z, 0) .
$$

Thence,

$\bar{u}=\frac{1}{\delta} \int_{0}^{\delta} u(r, \zeta, t) \mathrm{d} \zeta \equiv u_{b}-\frac{1}{\delta} \int_{0}^{\delta} \zeta u_{\zeta} d \zeta=u_{b}-\frac{1}{\delta} h_{r} H(\delta-h+Y) \int_{h-Y}^{\delta} \frac{\zeta(\zeta-h+Y) \mathrm{d} \zeta}{A(\Theta)}$,

with $H(x)$ the step function. However, for our illustrative viscosity law,

$$
A(\Theta)=\exp (\gamma(1-\Theta))=\exp \left(\gamma\left(1-\Theta_{b}\right)+q \mathscr{P}(\zeta / \delta)\right),
$$

where $q=\gamma\left(\Theta_{b}-\Theta_{s}\right)$. Hence, on specializing to $\mathscr{P}(\zeta / \delta)=(1-\zeta / \delta)^{2}$,

$$
\bar{u}=u_{b}-h_{r} y_{L}^{2} \delta^{2} \exp \left(-\gamma\left(1-\Theta_{b}\right)\right)\left[I_{0}-\left(1+y_{L}\right) I_{1}+y_{L} I_{2}\right],
$$

where $y_{L}=\operatorname{Max}[1-(h-Y) / \delta, 0]$ and

$$
I_{j}=\int_{0}^{1} y^{j} \exp \left(-q y_{L}^{2} y^{2}\right) \mathrm{d} y .
$$

These integrals can be written as error functions for $j$ even, or performed analytically for $j$ odd.

Similarly, we find

$$
\overline{u \Theta}-\bar{u} \bar{\Theta}=\frac{y_{L}^{3} \delta^{2} h_{r}}{3} \exp \left(-\gamma\left(1-\Theta_{b}\right)\right)\left(\Theta_{b}-\Theta_{s}\right)\left[I_{1}-I_{2}+y_{L}^{2}\left(I_{4}-I_{3}\right)\right] .
$$

We now have a fully prescribed skin model. 


\subsection{A summary of the equations}

For $\mathscr{P}(x)=(1-x)^{2}, \alpha\left(\Theta_{s}\right)=\Theta_{s}$ and $\mu^{2}=\kappa$, the equations are summarized as follows:

$$
h_{t}=-\frac{1}{r} \frac{\partial}{\partial r}[r(\mathscr{U}+\delta \bar{u})]+\Omega, \quad \mathscr{E}_{t}+\frac{1}{r} \frac{\partial}{\partial r}[r(\bar{u} \mathscr{E}-\mathscr{G})]=\frac{3}{2} \Theta_{s},
$$

with

$$
\begin{aligned}
& \Theta_{s}= \begin{cases}1+\mathscr{E} / 2-\sqrt{\mathscr{E}(1+\mathscr{E} / 4)} & \mathscr{E} \leqslant \mathscr{E}_{m}=h^{2} /[2 \sqrt{\kappa}(h+2 \sqrt{\kappa})], \\
(\sqrt{\kappa} / h)(3 h-2 \sqrt{\kappa} \mathscr{E}) /(h+3 \sqrt{\kappa}) & \mathscr{E}>\mathscr{E}_{m},\end{cases} \\
& \delta=\left\{\begin{array}{ll}
2 \sqrt{\kappa}\left(1-\Theta_{s}\right) / \Theta_{s} & \mathscr{E}^{*} \leqslant \mathscr{E}_{m}, \\
h, & \mathscr{E}>\mathscr{E}_{m},
\end{array} \quad \Theta_{b}= \begin{cases}1 & \mathscr{E} \leqslant \mathscr{E}_{m}, \\
(h+2 \sqrt{\kappa}) \Theta_{s} / 2 \sqrt{\kappa} & \mathscr{E}>\mathscr{E}_{m},\end{cases} \right. \\
& u_{b}=-\frac{1}{2} h_{r} Z(2 Y-Z), \quad \mathscr{U}=(h-\delta) u_{b}-\frac{1}{6} h_{r} Z^{2}(2 Z-3 Y), \\
& \bar{u}=u_{b}-\frac{\delta^{2} h_{r}}{2 q} \exp \left(-\gamma\left(1-\Theta_{b}\right)\right)\left[\left(2 q y_{L}+1\right) y_{L} I_{0}-1-y_{L}+\exp \left(-q y_{L}^{2}\right)\right], \\
& q=\gamma\left(\Theta_{b}-\Theta_{s}\right) \text {, } \\
& \mathscr{G}=y_{L} \delta^{3} h_{r} \exp \left(-\gamma\left(1-\Theta_{b}\right)\right) \frac{2 q\left(1-I_{0}\right)+3 I_{0}-\exp \left(-q y_{L}^{2}\right)-2}{8 \gamma q \sqrt{\kappa}}, \\
& I_{0}=\frac{\sqrt{\pi} \operatorname{Erf}\left(y_{L} \sqrt{q}\right)}{2 y_{L} \sqrt{q}}, \\
& Y=\operatorname{Max}\left(h-\frac{B}{\left|h_{r}\right|}, 0\right), \quad Z=\operatorname{Min}(Y, h-\delta), \quad y_{L}=\operatorname{Max}\left(1-\frac{h-Y}{\delta}, 0\right) \text {. }
\end{aligned}
$$

\subsection{Some limits}

If $Y<h-\delta, y_{L}=0$ and the fake yield surface lies inside the yielding region. The temperature change then has no effect, reflecting how rheological changes are unimportant within the pseudo-plug. Cooling affects the spreading dynamics only when the skin extends into the shearing regions, i.e. when $h-\delta<Y$.

If we ignore the motion of the dome, the heat equation reduces to $\mathscr{E}_{t}=3 \Theta_{s} / 2$. Provided $\Theta_{s} \ll 1$, we integrate to find $\delta=2 \sqrt{3 \kappa t}$, an estimate we use in the dimensional analysis of $\S 2.3 .1$.

$\gamma \rightarrow 0: \delta \bar{u}+\mathscr{U} \rightarrow-h_{r} Y^{2}(3 h-Y) / 6$, and the heat equation decouples, leaving the isothermal $h$-equation.

$\kappa \gg 1: \delta \rightarrow h, \Theta_{b} \rightarrow \Theta_{s}$, and we recover the vertically isothermal model in (2.16) with $\check{\Theta} \rightarrow \Theta_{s}$ and $\mathscr{U} \rightarrow \delta \bar{u}$.

$\gamma \gg 1: q \gg 1$ and $I_{0} \rightarrow \sqrt{\pi /\left(4 q y_{L}^{2}\right)} \ll 1$, implying that $\mathscr{G} \ll 1$ and $\bar{u} \rightarrow u_{b}$. Thus the flow in the skin becomes plug-like no matter where the fake yield surface lies. In other words, the enhanced vertical shear stress flattens the velocity profile inside the skin.

\subsection{Numerical solutions and comparison with shallow-lava theory}

We solve the skin equations using two codes (the comparison provides independent verification of the results); details are given in Appendix A.1. A sample solution of the skin model is displayed in figure 9 for $B=0, \kappa=0.01$ and $\gamma=6$, and corresponds to the computation with the full thin-layer model shown earlier in the first column of figure 1 and in panel $(a)$ of figures 2 and 4. Also displayed in figure 9 are the final profiles of thickness and surface and basal temperatures for both the full thin-layer theory and 
(a)

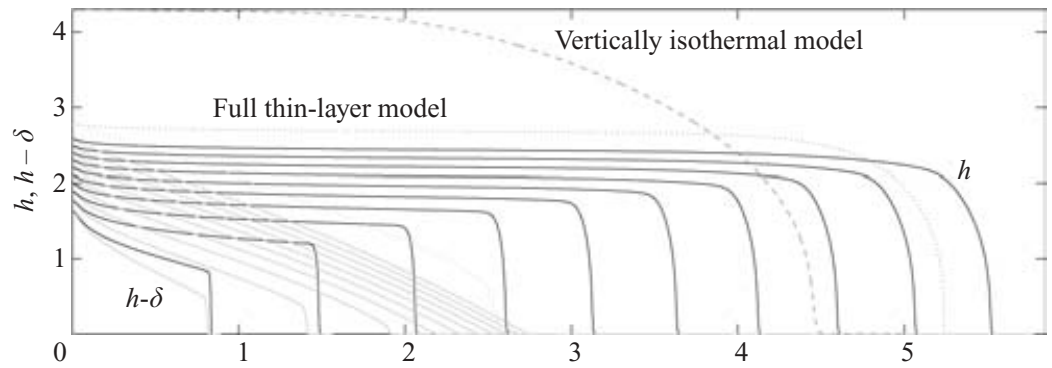

(b)

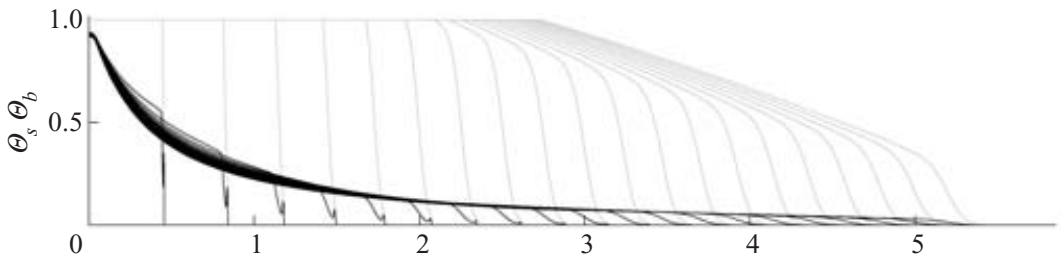

(c)

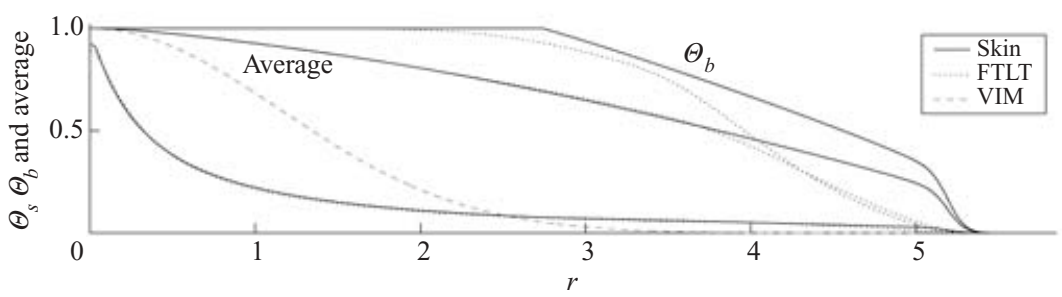

FIGURE 9. A solution of the skin model with $B=0, \kappa=0.01$. The panels show snapshots of (a) thickness (dark lines) and skin depth $h-\delta$ (light lines), and (b) surface (dark lines) and basal (light lines) temperature at $t=2.2 n^{2}, n=1, \ldots, 10$. In $(a)$, we also show the final thickness profiles of the full thin-layer theory (FTLT) and the vertically isothermal model (VIM), together the $\Theta=0.95$ isotherm of the full theory (light dotted line; this isotherm provides an estimate of skin depth). Panel (c) compares the final temperature distributions of the three models (namely the surface, basal and vertically averaged temperatures). The surface temperatures of the skin model and full thin-layer theory are barely distinguishable, whereas the vertically isothermal model only provides a prediction of the average.

the vertically isothermal model. The thickness profile and surface temperature of the skin model match those of the full thin-layer theory fairly closely. The comparison of basal temperature is less satisfactory for two reasons. First, the switch between the two forms of the integral-balance ansatz arising when the skin touches the base creates an artificial, abrupt change in $\Theta_{b}$. Secondly, in the full shallow-lava model, cooled fluid is advected along the surface and overrun by the evolving dome, forming the cold collar at the base. This collar of more viscous fluid impedes expansion, and is not captured by the skin theory (nor by the vertically isothermal model). As a result, the dome radius $R(t)$ is overestimated by the skin theory and the basal temperature profiles compare less favourably. Nevertheless, the skin theory is arguably adequate and certainly qualitatively correct. The large difference between $\Theta_{s}$ and $\Theta_{b}$ suggests that the vertically isothermal model is inadequate, and even the vertical average of $\Theta$ from the full theory compares poorly with the result from that model.

Figure 10 shows a selection of snapshots of solutions generated from the skin model, and should be compared with the results of the full thin-layer theory in figures 1 and 5. Figures 11 and 12 compare the predictions of both models for the evolution of the dome radius and height. Also included are corresponding results from the 

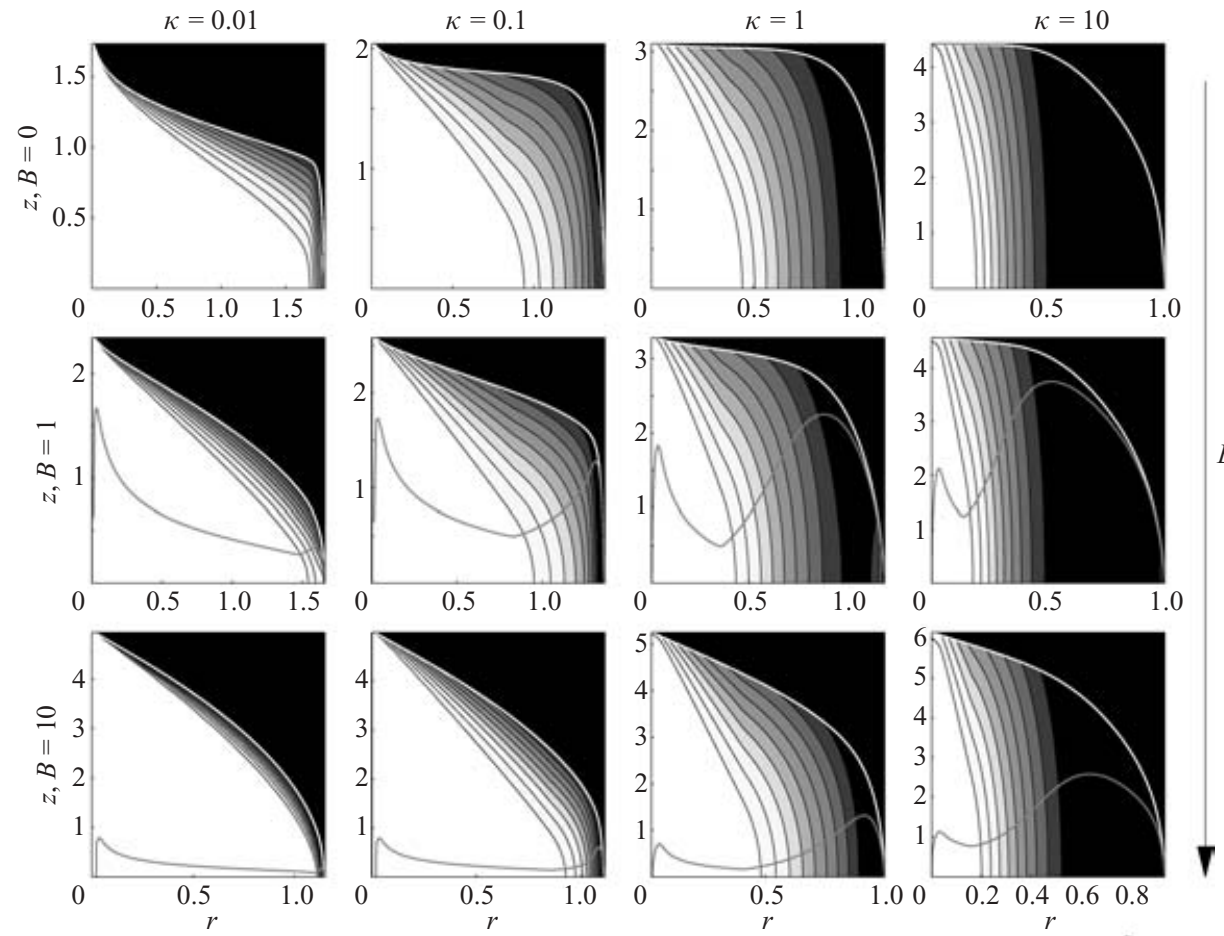

FiguRE 10. Snapshots of numerical solutions of the skin theory for $B=0,1$ and 10 , with $\kappa=0.01,0.1,1$ and 10 at $t=11$. The three rows should be compared with corresponding rows in figures 1 and 5 .

(a)

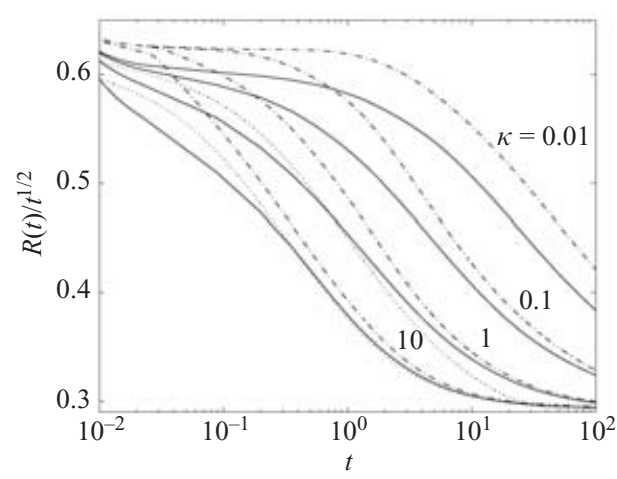

(b)

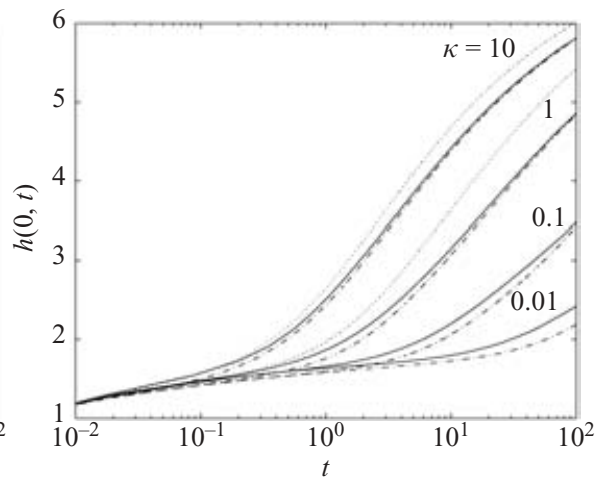

FiguRE 11. $(a)$ Dome radius and $(b)$ maximal height for the computations of figure 10 with $B=0$ and $\gamma=6$. The dashed lines show the skin model. The results of the full thin-layer model are represented by solid lines. The results of the vertically isothermal model are shown by dotted lines; only $\kappa=1$ and 10 are displayed since the others are too discordant to display.

vertically isothermal model. The temperature and height profiles are well captured by the skin theory over most values of the dimensionless diffusivity. The comparison improves as $\kappa$ increases; in the limit of large $\kappa$, the skin theory reduces to the standard lubrication model and both simplified theories adequately reproduce the full thermal 
(a)

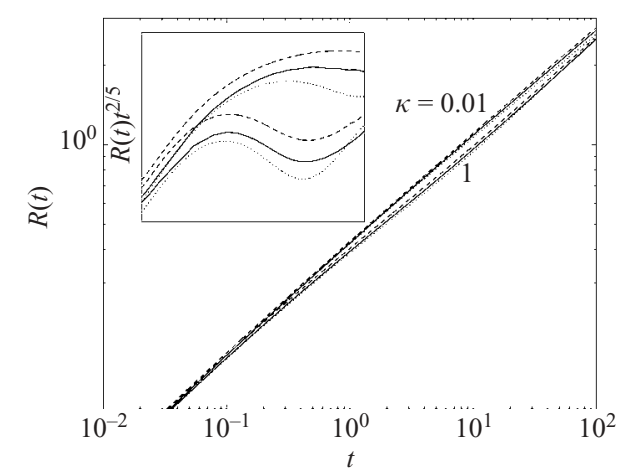

(b)

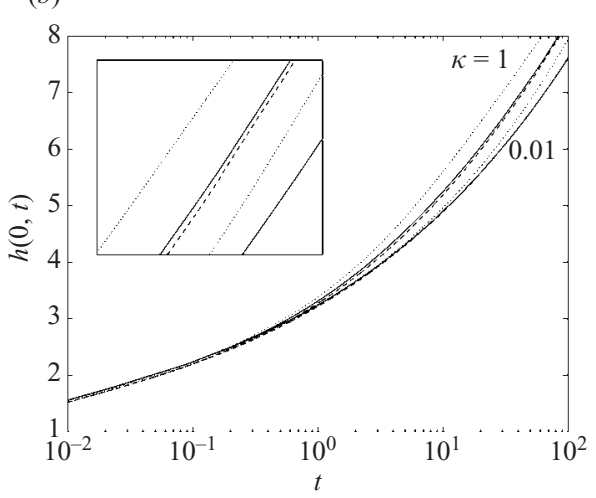

FIGURE 12. (a) Dome radius and $(b)$ maximal height for two of the computations of figure 10 with $\kappa=0.01$ and 1 , and $B=10$ and $\gamma=6$. The dashed lines show the skin model. The results of the full thin-layer model are represented by solid lines. The vertically isothermal model is represented by dotted lines. The inset in $(a)$ shows the data replotted as $R / t^{2 / 5}$; the inset in (b) shows a magnification near $t=100$. In $(b)$, the full thin-layer model and skin theory are indistinguishable.

evolution. The most substantial differences occur for small $\kappa$, which is where the collar of over-ridden cool material appears. The overturning at the edge is more prominent for larger values of $\gamma$ and smaller values of $B$, and so the skin theory becomes less accurate as $\gamma$ increases and $B$ decreases. Nevertheless, the dome radii and heights shown in figure 11 for $B=0$ capture the qualitative features of the dome evolution. Unsurprisingly, the vertically isothermal theory is much less satisfactory unless $\kappa$ exceeds 10 or so. (The curves predicted by that theory for $\kappa=0.01$ and 0.1 bear so little relation to those of the full thin-layer model that they confuse the picture and so have been omitted from figure 11.) The comparison amongst the three models is better in figure 12, which shows two representative sets of radius and height scalings for $B=10$, because thermal effects are masked by the pseudo-plug.

\section{Extensional stresses}

In experimental and geological flows, we often observe a thin skin of cooled material in which the viscosity is orders of magnitude larger than in the hot interior, and which plays a role in the flow dynamics. The shallow lava theory described in the earlier sections predicts the creation of such surface thermal boundary layers when the dimensionless diffusivity $\kappa$ is small. However, there is also little vertical shear in the velocity field within the skin when it is thin. Consequently, in that theory, rheological change has little effect, and the dome expands like an isothermal fluid at the eruption temperature, which contrasts sharply with the observations. The problem is that the shallow-lava analysis ignores the extensional viscous stresses (embodied in $\tau_{r r}, \tau_{\theta \theta}$ and $\tau_{z z}$ ) which provide extra resistance to expansion. In this section, we extend the theory by adding these stresses.

\subsection{The velocity field below the skin}

As before, the interior is characterized by $p=P_{s}+h-z$ and $\tau_{r z}=\tau_{s}-\left(P_{s r}+h_{r}\right)(h-z)$. Now, however, because of the overlying skin, we cannot directly impose the surface boundary conditions on this solution to eliminate $P_{s}$ and $\tau_{s}$, but we must match to a skin solution instead. As it turns out, $P_{s}=0$, which we assume herein, but $\tau_{s}$ does 
not disappear owing to the extensional stresses. This surface shear stress can have a dramatic effect on the velocity field even below the skin, as we now indicate.

The constitutive model provides the relation,

$$
\tau_{r z}=\tau_{s}-h_{r}(h-z)=u_{z}+B \operatorname{sgn}\left(u_{z}\right),
$$

provided $\left|\tau_{r z}\right| \equiv\left|h_{r}(h-z)-\tau_{s}\right|>B$, a condition that can be written in the alternative forms,

$$
z<Y_{L}=h-\frac{\tau_{s}}{h_{r}}-\frac{B}{\left|h_{r}\right|} \quad \text { or } \quad z>Y_{U}=h-\frac{\tau_{s}}{h_{r}}+\frac{B}{\left|h_{r}\right|} .
$$

The surface $z=Y_{L}$ is related to the fake yield surface, $Y$, of previous sections. However, the effective surface stress, $\tau_{s}$, introduces a second fake yield surface, $z=Y_{U}$, which can lie inside the fluid. In this circumstance, a yielding region appears just beneath the surface, in addition to that beside the base. The upper yielding region is driven by the stress induced in the skin as the interior flows beneath.

The lower yielding region exists if $Y_{L}>0$, and the upper one if $h-\delta>Y_{U}$. For $0<Y_{L}<Y_{U}<h-\delta$, a pseudo-plug is contained in the region, $\left[Y_{L}, Y_{U}\right.$ ] and the velocity field is then given by

$$
u=\left\{\begin{array}{ll}
-h_{r}\left(2 Y_{L}-z\right) z / 2 & Y_{L}>z, \\
u_{p} & Y_{U}>z>Y_{L}, \\
u_{p}+h_{r}\left(z-Y_{U}\right)^{2} / 2 & h-\delta>z>Y_{U},
\end{array} u_{p}=-\frac{1}{2} h_{r} Y_{L}^{2},\right.
$$

where $u_{p}$ is the pseudo-plug radial velocity. When $Y_{L}<0$, the lower yielding region does not exist and the pseudo-plug is stationary, but the upper region can still flow under the action of the surface stress if $0<Y_{U}<h-\delta$. For $Y_{U}>h-\delta$ there is no upper yield region and the configuration is much as before.

For all the possible cases, the velocity at the base of the skin, $u_{b}$, and the depthintegrated radial velocity in the interior, $\mathscr{U}$, can be compactly written in the form,

$$
u_{b}=-\frac{1}{2} h_{r}\left[Y_{L+}^{2}+\left(-Y_{U}\right)_{+}^{2}-\left(Y_{L}-h+\delta\right)_{+}^{2}-\left(h-\delta-Y_{U}\right)_{+}^{2}\right]
$$

and

$$
\mathscr{U}=-\frac{1}{6} h_{r}\left[Y_{L+}^{2}\left(3 h-3 \delta-Y_{L}\right)+\left(-Y_{U}\right)_{+}^{2}\left(3 h-3 \delta-Y_{U}\right)+\left(Y_{L}-h+\delta\right)_{+}^{3}-\left(h-\delta-Y_{U}\right)_{+}^{3}\right] .
$$

\subsection{In the skin}

Within the skin, we assume that cooling creates a sufficiently strong rheological change to promote the longitudinal stresses, $\tau_{r r}$ and $\tau_{z z}$, to lower order in $\epsilon$. In our formulation, the primary changes occur in the viscosity, and so we set $A(\Theta)=\epsilon^{-2} a(\Theta)$, with $a(\Theta)$ a rapidly decreasing, but order one function such that $a \rightarrow O\left(\epsilon^{2}\right)$ for $\Theta \rightarrow 1$. This increase of viscosity is sufficient to bring the extensional stresses into the main balance, but can also enhance the vertical shear stresses. However, $\tau_{r z} \sim A u_{z} \equiv \epsilon^{-2} a u_{z}$ must remain order unity in order to match the stresses in the interior and to balance the gravitational driving force. Thus, $u_{z}$ must decrease to counter any increase in viscosity, indicating that the radial velocity becomes nearly uniform over the skin, as in the simpler theory of the previous section. This motivates the expansion,

$$
\left.\begin{array}{l}
u(r, z, t)=u_{b}(r, t)+\epsilon^{2} u_{\Theta}(r, z, t), \\
w(r, z, t)=w_{b}(r, t)+\left(r u_{b}\right)_{r}(h-\delta-z) / r+\epsilon^{2} w_{\Theta}(r, z, t),
\end{array}\right\}
$$

where $\left(u_{b}, w_{b}\right)$ is the velocity field at the base of the skin. 
In accord with (5.6), we now set

$$
\epsilon \tau_{r r}=\hat{\tau}_{r r}=2 a \frac{\partial u_{b}}{\partial r}, \quad \epsilon \tau_{\theta \theta}=\hat{\tau}_{\theta \theta}=\frac{2}{r} a u_{b}, \quad \epsilon \tau_{z z}=\hat{\tau}_{z z}=2 a w_{z},
$$

but leave $\tau_{r z}=a\left(\partial u_{\Theta} / \partial z+w_{r}\right)$ order one. (The temperature is assumed not to affect the yield stresses, and so they disappear from the leading order in the skin.) The momentum equations become

$$
p_{r}=\partial_{r} \hat{\tau}_{r r}+\partial_{z} \tau_{r z}+\frac{1}{r}\left(\hat{\tau}_{r r}-\hat{\tau}_{\theta \theta}\right), \quad p_{z}=-1+\partial_{z} \hat{\tau}_{z z}
$$

on which we impose the mechanical boundary conditions,

$$
p-\hat{\tau}_{z z}=\tau_{r z}+h_{r}\left(p-\hat{\tau}_{r r}\right)=0 \quad \text { at } z=h .
$$

We first find

$$
p-\hat{\tau}_{z z}=h-z
$$

For $h-z>\delta, a \rightarrow 0$ and so $p \rightarrow h-z$. The match with the interior solution therefore demands $P_{s}=0$, as we already assumed. Now we focus on the radial momentum equation, using the pressure field (5.10):

$$
\frac{\partial \tau_{r z}}{\partial z}=h_{r}-4 \frac{\partial}{\partial r}\left[\frac{a}{r} \frac{\partial}{\partial r}\left(r u_{b}\right)\right]+\frac{2 u_{b}}{r} \frac{\partial a}{\partial r} .
$$

The integral of (5.11) yields the relation,

$$
\tau_{r z}=-h_{r}(h-z)+4 \frac{\partial}{\partial r}\left[\frac{1}{r} \frac{\partial\left(r u_{b}\right)}{\partial r} \int_{z}^{h} a(\Theta) \mathrm{d} z\right]-2 \frac{u_{b}}{r} \frac{\partial}{\partial r}\left[\int_{z}^{h} a(\Theta) \mathrm{d} z\right] .
$$

We now take $z=h-\delta$ and match with the interior solution, to find:

$$
\tau_{s}=4 \frac{\partial}{\partial r}\left[\frac{\delta \bar{a}}{r} \frac{\partial}{\partial r}\left(r u_{b}\right)\right]-2 \frac{u_{b}}{r}(\delta \bar{a})_{r}
$$

where

$$
\bar{a}=\frac{1}{\delta} \int_{h-\delta}^{h} a(\Theta) \mathrm{d} z=\int_{0}^{1} a\left[1-\left(1-\Theta_{s}\right) \mathscr{P}(x)\right] \mathrm{d} x
$$

denotes the skin-averaged viscosity. For example, with our illustrative viscosity law, $A(\Theta)=\exp (\gamma(1-\Theta)) \equiv \epsilon^{-2} a(\Theta)$, we obtain

$$
\begin{aligned}
\bar{a}\left(\Theta_{s}\right)=\epsilon^{2} \exp (\gamma(1- & \left.\left.\Theta_{b}\right)\right) \int_{0}^{1} e^{q y^{2}} \mathrm{~d} y \approx \epsilon^{2} \exp \left(\gamma\left(1-\Theta_{s}\right)\right) \\
& \times \int_{0}^{1} e^{-2 q y} \mathrm{~d} y=\epsilon^{2} \exp \left(\gamma\left(1-\Theta_{s}\right)\right) \frac{1-\exp (-2 q)}{2 q} .
\end{aligned}
$$

\subsection{The extensional stress model}

The model equations can now be summarized as follows. Because there is little shear inside the skin, $\bar{u} \rightarrow u_{b}$. Hence, the total mass flux is $\mathscr{U}+\delta u_{b}$, and $\mathscr{E}$ is advected only by $u_{b}$. Thus,

$$
h_{t}=\frac{1}{r} \frac{\partial}{\partial r}[r(\mathscr{U}+\delta \bar{u})]+\Omega, \quad \mathscr{E}_{t}+\frac{1}{r} \frac{\partial}{\partial r}[r(\bar{u} \mathscr{E}-\mathscr{G})]=\frac{3}{2} \Theta_{s},
$$

with $\bar{u}=u_{b}$ and $\mathscr{G}=0$. The relation between $\mathscr{E}$ and $\Theta_{s}$ is the same as before.

The expressions (5.4) and (5.5) give $u_{b}$ and $\mathscr{U}$ in terms of $\tau_{s}$, which is in turn given by (5.13). Equivalently, we may eliminate $\tau_{s}$ from these formulae to find a differential 
equation for $u_{b}$, which therefore plays the role of a third dependent variable in the extensional stress model. Convenient formulae for $Y_{L}, Y_{U}$ and $\tau_{s}$ can be written down that ease this passage. However, for brevity and because we only present numerical solutions for this simpler problem, we quote the full equations for $B=0$ :

$$
4 \frac{\partial}{\partial r}\left[\frac{\delta \bar{a}}{r} \frac{\partial}{\partial r}\left(r u_{b}\right)\right]=u_{b}\left[(h-\delta)_{+}^{-1}+\frac{2}{r}(\delta \bar{a})_{r}\right]+\frac{1}{2} h_{r}(h+\delta),
$$

with

$$
\begin{gathered}
\mathscr{U}=\frac{1}{2}(h-\delta)_{+} u_{b}-\frac{1}{12} h_{r}(h-\delta)_{+}^{3}, \\
\bar{a}=a_{0} \exp \left(\gamma\left(1-\Theta_{b}\right)\right) \frac{\sinh \gamma\left(\Theta_{b}-\Theta_{s}\right)}{\delta \Theta_{s} \sinh \gamma}, \quad a_{0}=\frac{2 \epsilon^{2} \sqrt{\kappa}}{\gamma} \sinh \gamma .
\end{gathered}
$$

5.4. An amalgamation of the two skin models

Through $\bar{a}, \epsilon^{2}$ appears explicitly as a parameter. The extensional stress theory is set out for $\epsilon^{2} e^{\gamma} \sqrt{\kappa} \sim \gamma$. If $\epsilon^{2} e^{\gamma} \sqrt{\kappa} \ll \gamma$, the extensional viscosity disappears from the skin, and we would hope to recover the earlier skin theory. As the extensional stress theory stands, however, the radial velocity is always uniform in the skin, and any vertical shear appears in the higher-order correction, $\epsilon^{2} u_{\Theta}$, which has been dropped. Worse still, when $\delta=h$, the theory predicts that the dome must become stationary owing to the enhanced vertical shear stress. However, the dome should still expand, albeit at the much lower rate dictated by the rheology of the cold fluid.

To connect the two theories and allow expansion for $\delta=h$, we add the mass flux expected for $\bar{a} \rightarrow 0$ to the extensional stress model. More specifically, in (5.16), we introduce the relations,

$$
\begin{gathered}
\bar{u}=u_{b}-\frac{\delta^{2} h_{r}}{2 q} \exp \left(-\gamma\left(1-\Theta_{b}\right)\right)\left[(2 q+1) I_{0}-2+\exp (-q)\right], \\
\mathscr{G}=\delta^{3} h_{r} \exp \left(-\gamma\left(1-\Theta_{b}\right)\right)\left[\frac{2 q\left(1-I_{0}\right)+3 I_{0}-\exp (-q)-2}{8 \gamma q \sqrt{\kappa}}\right]
\end{gathered}
$$

(cf. (4.22) and (4.23), with $y_{L}=1$ ), which gives our final combined set of model equations. The extra terms in (5.20) and (5.21) are relatively small in the limit relevant to the extensional stress model, but are essential in allowing further expansion of the dome when the skin extends to the fluid base.

\subsection{Numerical solutions}

\subsubsection{Pure extensional stress}

Sample numerical solutions of (5.16)-(5.21) are shown in figures 13-15. These show cases in which the skin thickness $\delta$ is assumed negligible in comparison to $h$, which leaves only the effect of the extensional stresses, the strength of which we estimate using the parameter $a_{0}$. Figure 13 shows a case with relatively small stresses, and figure 14 displays a case with relatively strong ones. Figure 15 represents an intermediate value. These examples illustrate two special limits of the problem.

The weaker skin stress has little effect, and the fluid beneath expands like an isothermal flow at the eruption temperature (see figure 13). When the extensional stress is dominant, the skin brakes almost to a halt; the fluid flows underneath and gradually lifts up the skin, which remains more uniform in temperature (figure 14). There is little conveyance of material to the rim in this instance, and the fluid expands like a hot isothermal fluid with a surface on which $u=0$ so that the material can only 

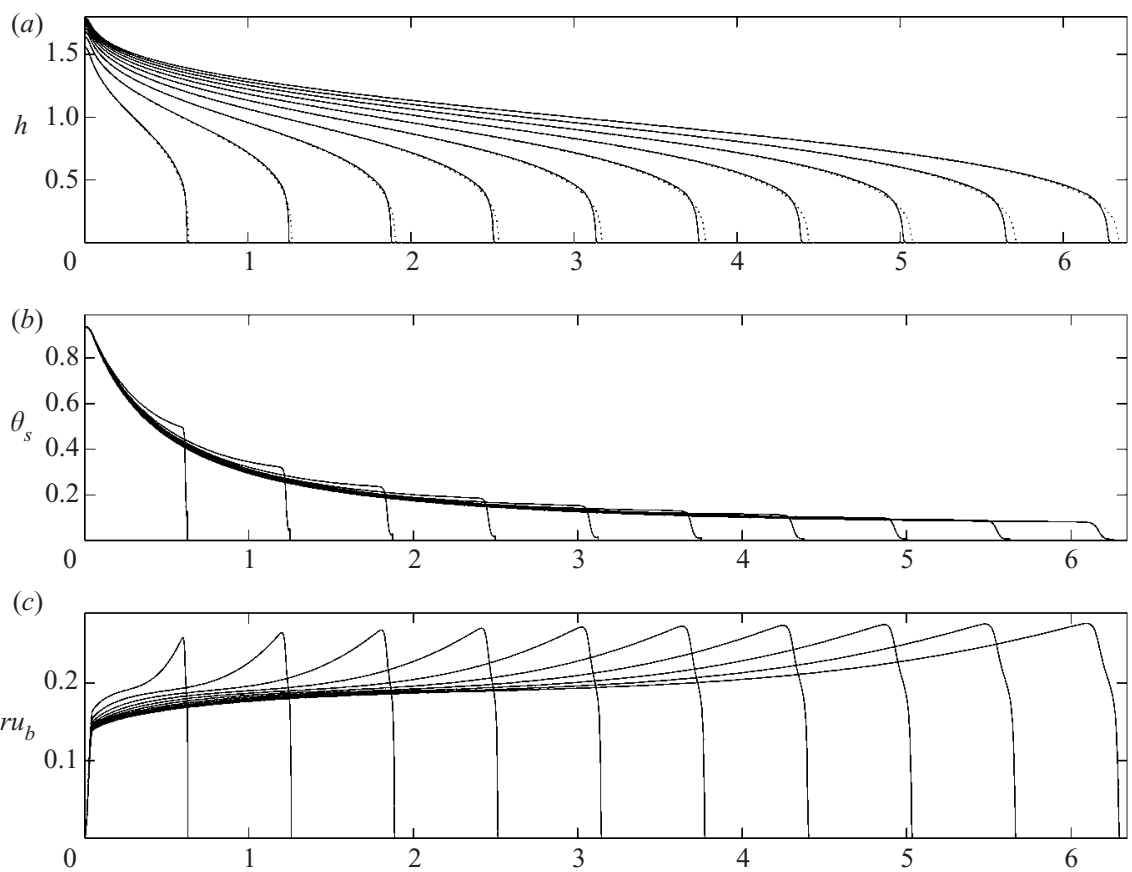

(d)

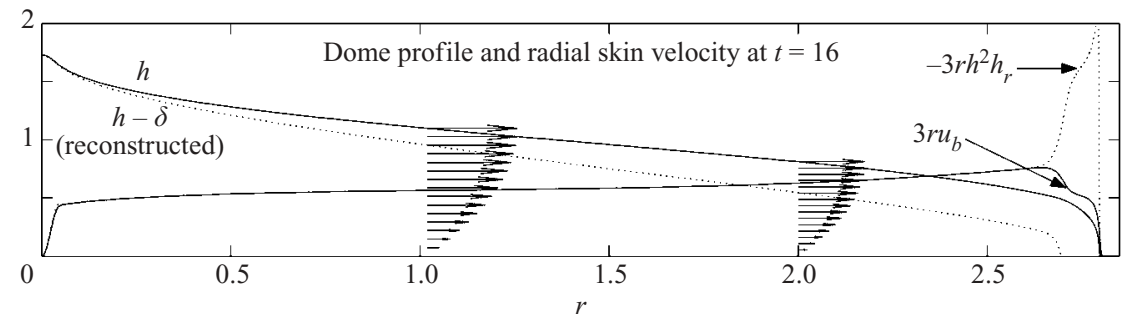

FIGURE 13. A simulation for a source with $B=0$, assuming the thermal boundary layer has negligible thickness $(\delta \ll h)$. $(a)-(c)$ show snapshots of the thickness, the surface temperature and $r u_{b}$ at the times $t=n^{2}, n=1,2, \ldots, 10$. The dotted lines in $(a)$ show the corresponding isothermal solution with a free surface. In this case, the surface viscosity is not large and the surface velocity field approaches $-h^{2} h_{r} / 2$ (the usual isothermal result) except for boundary layers at the source and near the edge, as shown in $(d)$. This picture also shows the extent of the skin at $t=16$, had it not been assumed infinitesimally thin (hence displaying a consistency check), and illustrates the vertical profile of the radial velocity. The parameters of the model are $a_{0}=10^{-4}$ and $\gamma=4$. The diffusion parameter $\kappa$ does not appear explicitly in the theory when $\delta$ is assumed much less than $h$; in $(d), \delta$ is reconstructed assuming $\kappa=10^{-3}$.

rise vertically (see dotted lines in the first panels, and the velocity profile in the final panels). $\uparrow$ The time history of the dome radius and height is shown in figure 16 . The convergence of the solutions to the two characteristic isothermal cases (zero surface

$\dagger$ This limit of strong extensional stress also arises with arbitrary $B$, and we find an analogous situation: provided $\delta$ remains much smaller than $h, u_{s} \rightarrow 0$, leaving an expanding dome with a surface that can only rise vertically. Underneath, the radial velocity profile resembles a Poiseuille flow of a viscoplastic fluid, with two yielding regions symmetrically placed around a pseudo-plug. Overall, in comparison with an expanding dome with a free surface, the mass flux is reduced by a factor of 4 , and the Bingham number is increased by a factor of 2 . 
(a)

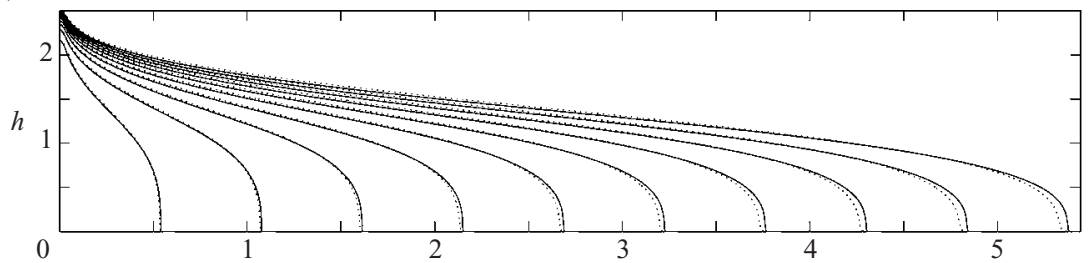

(b)

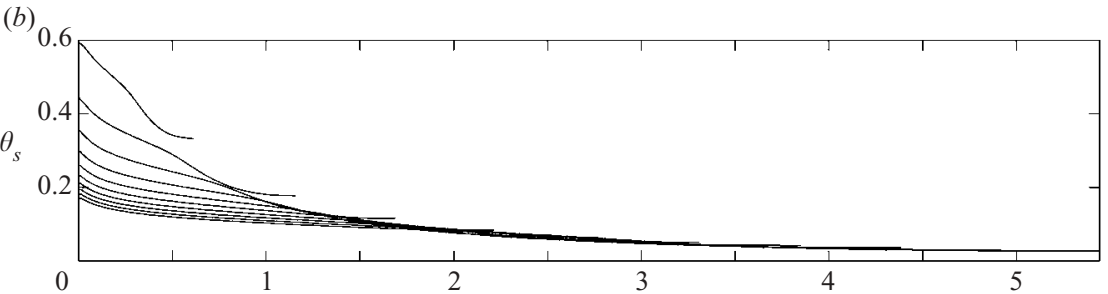

(c)

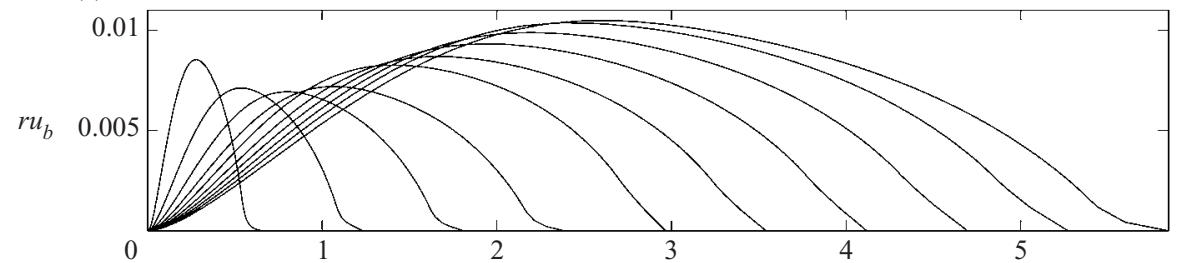

(d)

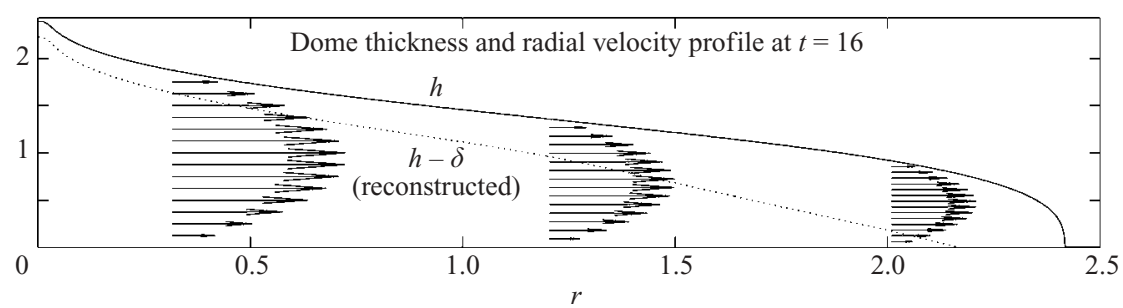

FIGURE 14. A simulation where the surface viscosity is relatively large and the velocity field in the skin becomes practically zero. $a_{0}=2$ and $\gamma=6 ; \delta$ is constructed assuming $\kappa=10^{-3}$, but is not included in the computation and here simply shows where the skin would have been as a consistency check. The dotted lines in $(a)$ show an isothermal simulation constrained to have a surface on which $u=0$ and can only be lifted vertically.

stress and a surface with $u=0$ ) is evident over longer times (in which $R \sim t^{1 / 2}$ and $\left.h(0, t) \sim t^{0}\right)$.

\subsubsection{Finite skin thickness}

Extrusions with relatively weak and strong extensional stresses, but finite skin depth, are shown in figures 17 and 18. For the relatively weak skin stress, because of the strong surface flow, a cooled edge layer quickly develops that significantly decelerates the fluid edge and builds up steep sides to the dome. Overall, the expansion is very similar to that of an extrusion with $a_{0}=0$. In fact, a good estimate of the expansion rate seems to be provided by dimensional analysis (the curve $D S$ in figure 17), assuming that the edge layer controls the radial expansion and the dimension of this layer grows like $2 \sqrt{3 t}$ (see $\S 4.1$ ). This conclusion is in agreement with observations by Stasiuk et al. (1993), drawn from their experiments with syrup. By contrast, the dome height $h(0, t)$, is not well predicted either by the cold dome or the dimensional analysis, both of which predict much thicker domes. This arises because the central 
(a)

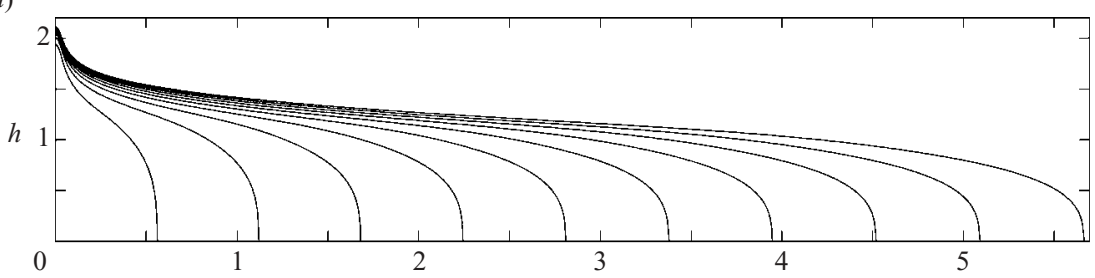

(b)

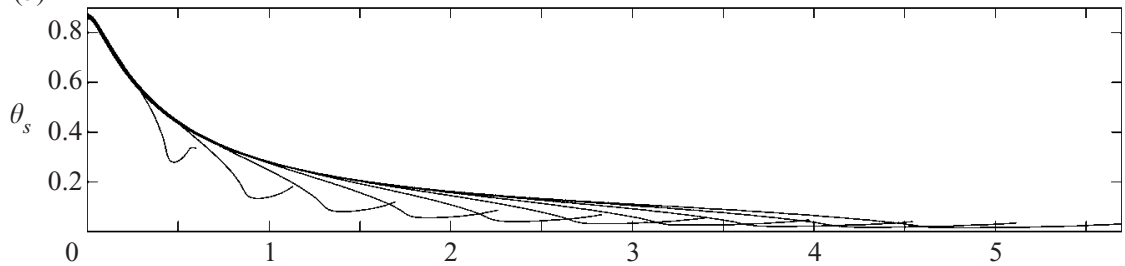

(c)

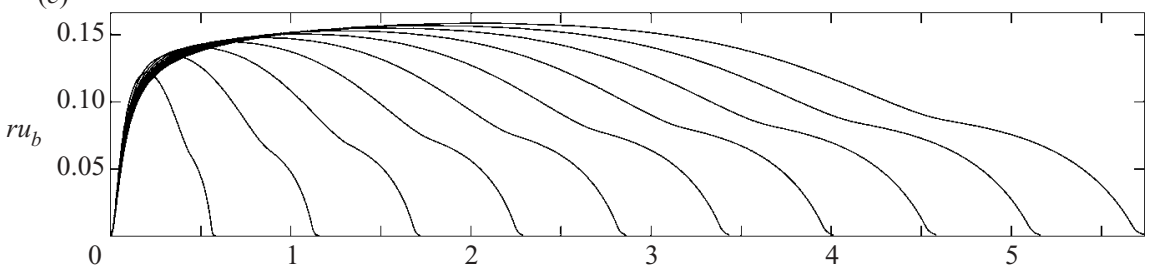

(d)

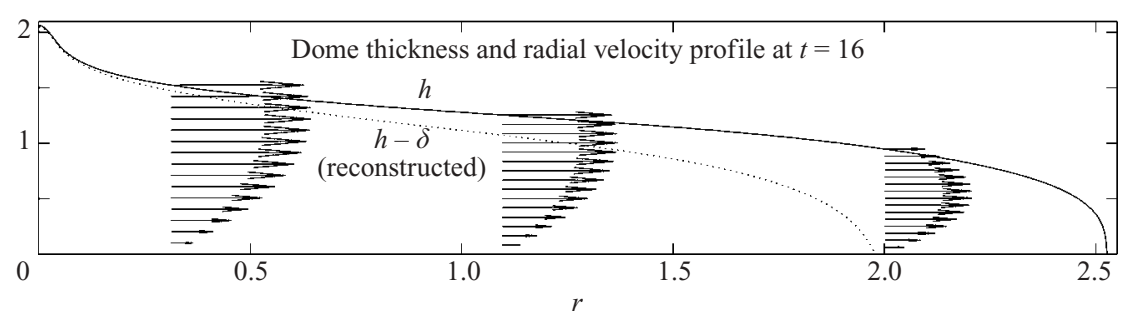

FIGURE 15. Sample solution for a source with intermediate skin strength. $(a)-(d)$ as in figures 13-14. $a_{0}=0.05, \gamma=4$ and $\kappa=10^{-3}$.
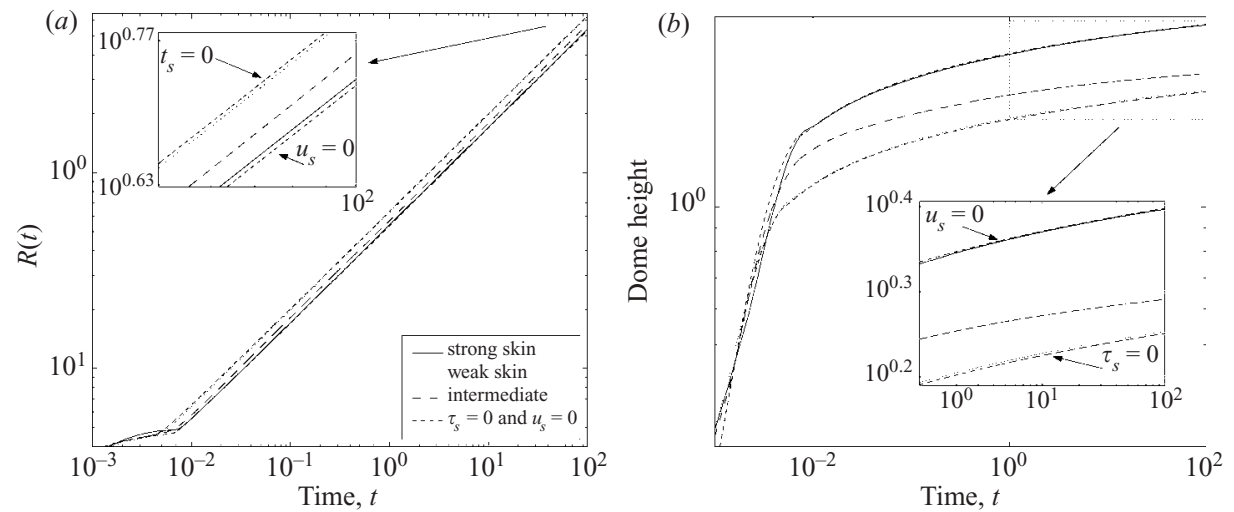

FIgURE 16. (a) Radius and $(b)$ dome heights against time for the three sample extrusions (weak, intermediate and strong extensional stress). The dashed lines show isothermal extrusions, without extensional stresses, and with a viscosity given by the eruption temperature; the two isothermal cases differ in having either a free surface $\left(\tau_{s}=0\right)$, or a surface with $u=0$ at $z=h$ $\left(u_{s}=0\right)$. 

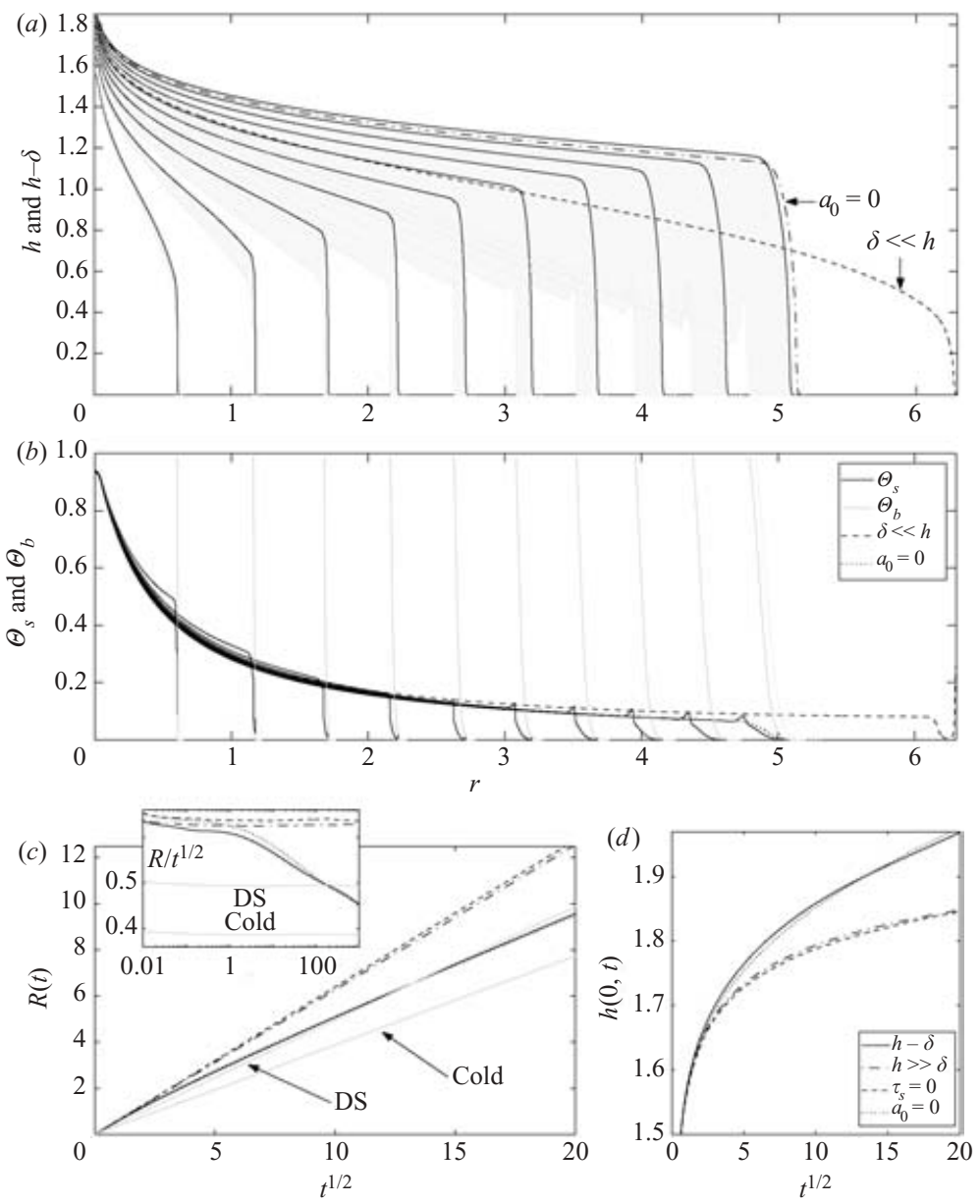

FiguRE 17. An extrusion with small extensional stress and finite skin thickness. (a) Snapshots of the dome surface and skin for $t=n^{2}$ and $n=1,2, \ldots, 10$; the shaded region shows the skin. The dashed curve shows the final height profile if the skin thickness were assumed infinitely thin, and the dash-dotted line shows an extrusion assuming $a_{0}=0 .(b)$ shows corresponding surface and basal temperatures, $\Theta_{s}$ and $\Theta_{b} .(c, d)$ show the dome radius and maximal height against time. Also displayed are the radius and height for an isothermal extrusion with stress-free surface (labelled $\tau_{s}=0$ ), the extrusion with $a_{0}=0$, an extrusion with a cooling skin assuming $\delta \ll h$. In $(c)$, showing the evolution of the dome radius, we have added two extra curves (by light solid lines); these show the radius of a dome expanding isothermally at the ambient temperature ('Cold'), and the prediction expected from dimensional scaling ('DS'), assuming that a slender edge controls the expansion (see $\S 4.1) . \kappa=10^{-3}, \gamma=4, a_{0}=10^{-4}$.

parts of the dome are much flatter than expected if we were to extrapolate from the cooled edge.

The success of the dimensional scaling result in figure $17(a)$ is surprising because it assumes that the edge layer grows in $r$ like a motionless boundary layer, yet there is no radial diffusion of heat in the theoretical model. In fact, the result should be viewed with some caution; although the trend in the picture seems to be well predicted, a closer examination (namely a plot of $R / \sqrt{t}$, as in the inset) shows a result much like that found for the models without extensional stresses: the radial expansion progresses through a transition between the extremes dictated by the hot and cold viscosities 

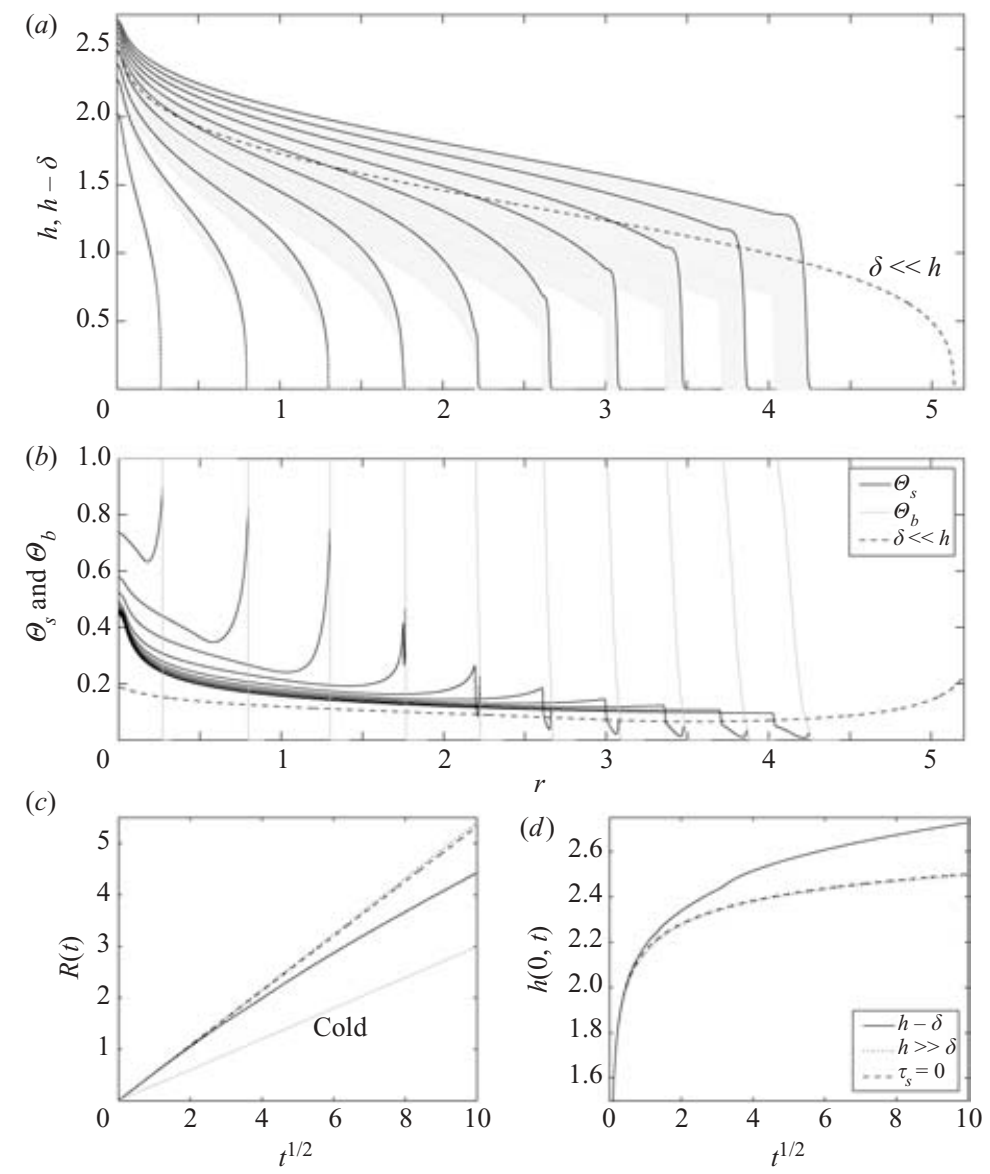

FIGURE 18. A similar picture to figure 17 showing an extrusion with a strong extensional stress and finite skin thickness. In this case, neither the extrusion with $a_{0}=0$ nor the dimensional scaling result are shown. $\kappa=10^{-3}, \gamma=4, a_{0}=2$.

(see figure 3). Figure 17(a) shows the radial expansion over the transitional range, where the dome radius by chance looks similar to that predicted by dimensional analysis. However, the detailed dependence on time is not well predicted and $R(t) / \sqrt{t}$ gradually descends to follow the cold expansion rate.

In the case with a strong extensional stress, the skin deepens more uniformly. At least initially, the reduced surface flux leaves a much thinner cooled edge layer at the flow front, and the dome expands like an isothermal dome with zero radial velocity at the surface. However, an edge layer eventually develops, steepening the sides of the dome and braking the dome's expansion. The expansion is then closer to that expected for a cold isothermal dome without extensional stresses.

\section{Discussion}

Lubrication-style approximations for spreading cooling flows run into complications when thermal diffusion is relatively slow. In this circumstance, the asymptotic reduction fails to simplify the heat equation, and in the thin-layer model we couple this unsimplified equation to a nonlinear diffusion equation for the fluid thickness. 
Part of our aim in this work has been to present an efficient numerical scheme for tackling this type of thin-layer model. We have also offered two simpler models, a vertically isothermal theory and a skin theory based on the integral-balance method, that can give adequate results in some limits of the problem.

The full thin-layer model captures much of what appears in experiments with cooling syrup (Stasiuk et al. 1993); we see a steepening in the flow front over time, advection of cooled fluid along the surface to the flow front, a collar of overridden cool fluid, and a plateau developing behind the steepened front (at least for small effective diffusivity and over short enough times). For a fluid without yield stress, dimensional scaling analysis suggests that the dome radius should vary as $R(t) \sim C t^{1 / 2}$ where the constant $C$ proceeds in time through the sequence: $1 \rightarrow\left(\kappa A_{0}\right)^{-1 / 7} \rightarrow A_{0}^{-1 / 8}$, where $A_{0}$ is the factor by which the viscosity is enhanced on cooling and $\kappa$ the dimensionless conductivity. In numerical computations with both the full model and with the two simpler models, we certainly observe the first and last scaling; the dome begins to expand as though it were entirely hot, but much later expands as though it were completely cold. The intermediate scaling is not truly observed, although there is some suggestion that this scaling may superficially resemble the correct radial expansion, as seen in experiments. The intermediate scaling is not observed because the cooled layer at the edge does not thicken like a motionless thermal boundary layer, but is fed by flow along the dome's surface. Moreover, the cold material does not collect at the edge, but is overridden to produce the underlying collar.

For a yield-stress fluid, there is a reduced sensitivity to thermal effects, as seen in the experiments by Griffiths \& Fink (1997). Cooling has little effect because of the almost plug-like flow that arises in the upper parts of the dome. Here, there is little vertical shear and any elevation in viscosity due to cooling is inconsequential. This shielding effect was observed in earlier models (paper 1).

Although such shielding is physically plausible, a key effect is missing: the promotion of extensional viscous stresses by cooling. The thin-layer model does not incorporate such stresses because, in the standard asymptotic scalings, they lie at a higher order than the vertical shear stress. Yet cooling-induced variations are very significant in fluids like lava, which often form rheologically distinct skins atop the flow. Extensional stresses are straightforwardly added to the skin theory, and so set the stage for study of these flows. The model predicts that significant extensional stresses bring the skin almost completely to rest, although hot fluid continues to expand underneath. Eventually, cooling thickens the skin to the point where it reaches the base, at which point vertical shear stresses again take control.

One future application of the current theory is to lava flows in which surface morphology is used to infer emplacement conditions (Griffiths 2000). For example, fingers and lobes appear in many extrusion experiments, and in paper 1 we concluded that axisymmetric expansion in the vertically isothermal model could be linearly unstable to some non-axisymmetric perturbations. Whether such perturbations remain unstable in more refined models with lower thermal diffusivity remains an open question. It is also worth noting that domes with strong extensional stresses can have a very thin skin near the flow front, which could be prone to rupture. Hot less viscous material may then emerge to create the complicated 'compound' flows visible in many lava fields.

We should also add that the temperature-dependence of the yield stress can be significant in lava, an effect that we have completely ignored so far. In this geological application, the yield stress arises from the crystal content, and crystallization occurs on cooling. To conclude, we show a solution of the shallow lava model with $\tau_{p}$ a prescribed, exponential function of $T$; see figure 19. In dimensionless variables, this 
(a)

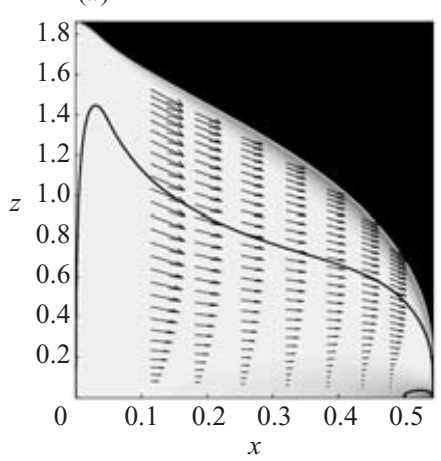

(b)

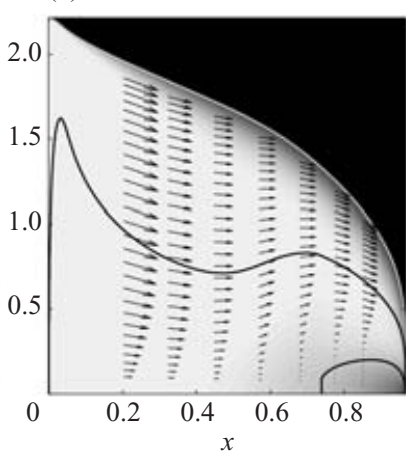

(c)

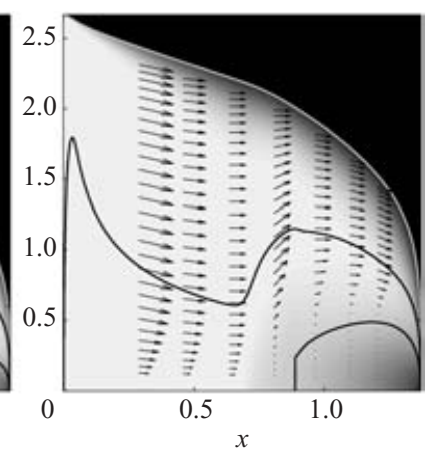

FIGURE 19. Snapshots of an evolving dome with a temperature-dependent yield stress at times $(a) t=1,(b) 4$ and $(c) 11$, of the temperature distribution (colourmap), the fluid surface (lighter lines) and fake yield surfaces (dark lines). The yield stress is given by $\tau_{p}(T)=$ $\tau_{p}\left(T_{e}\right) e^{\gamma_{B}(1-\Theta)}$, with $\gamma_{B}=6$ and $B=H \tau_{p}\left(T_{e}\right) /\left(\eta_{e} U\right)=1$. The other parameters were $\gamma=6$ and $\kappa=0.01$. More details of the numerical procedure are given in Appendix A.2.

modification amounts to replacing $B$ by $B g(\Theta)$, with $g(1)=1$. The main novelty introduced by the temperature variation is that the yield condition, $\tau(r, z, t)>B g[\Theta(r, z, t)]$, is no longer straightforward to solve for the fake yield surface. In fact, there can be more than one solution to

$$
\tau(r, Y, t)=B g[\Theta(r, Y, t)],
$$

which raises the possibility of multiple pseudo-plugs. Indeed, the numerical solution displays the development of a second pseudo-plug within the expanding cold collar at the fluid edge (which may even contain a true plug). This emplaces a further barrier to the outflow and a strong upward current develops to surmount the collar. Otherwise, the flow dynamics is not very different from the cases considered earlier. The formation of the second pseudo-plug requires a dominance of the change in yield stress over that of the viscosity, which is guaranteed by suitable choices of the parameters (see paper 1).

This work was supported by the National Science Foundation (Grant no. DMS 72521).

\section{Appendix A. Numerical scheme}

We apply the change of variables, $r=\xi R(t)$ and $z=\zeta h(\xi, t)$, where $R(t)$ is the contact radius, to furnish the new set of evolution equations:

$$
\begin{gathered}
h_{t}=\frac{1}{\xi} \partial_{\xi}\left[-\xi \frac{\psi(\xi, 1)}{R}\right]+\Omega+\frac{\xi R_{t}}{R} h_{\xi}, \\
\Theta_{t}=-\left(\frac{u-\xi R_{t}}{R}\right) \Theta_{\xi}-\frac{1}{h}\left[w-\zeta\left(h_{t}+u \frac{h_{\xi}}{R}-\frac{\xi h_{\xi} R_{t}}{R}\right)\right] \Theta_{\zeta}+\frac{\kappa}{h^{2}} \Theta_{\zeta \zeta}, \\
u(\xi, \zeta)=-\frac{h_{\xi} h^{2}}{R} \int_{0}^{\zeta} \Upsilon_{\zeta} \mathrm{d} \zeta^{\prime}, \quad w(\xi, \zeta)=-\frac{1}{\xi R} \partial_{\xi}(\xi \psi)+\frac{\zeta h_{\xi}}{R} u+\Omega, \\
\psi(\xi, \zeta)=-\frac{h_{\xi} h^{3}}{R} \int_{0}^{\zeta} \Upsilon \mathrm{d} \zeta^{\prime}, \quad \Upsilon_{\zeta}= \begin{cases}0 & \text { for } \zeta>\eta, \\
(\eta-\zeta) / \mathscr{A}(\Theta) & \text { for } \zeta \leqslant \eta,\end{cases}
\end{gathered}
$$


and $\eta=Y / h=1-R B /\left(h\left|h_{\xi}\right|\right)$ is the yield surface in the rescaled domain. The boundary conditions are: $\Theta_{\xi}=h_{\xi}=0$ for $\xi=0$ and $\Theta_{\xi}=0, h=10^{-4}$ for $\xi=1 ; \Theta_{\zeta}=0$ at the bottom, outside the vent, $\Theta=1$ over the vent, and $\sqrt{\kappa} \Theta_{\zeta}=-h \Theta$ at the free surface.

We solve these equations by discretizing all the spatial derivatives, and then numerically integrating the large system of ordinary differential equations that result. We index the grid points by the pair of integers $(i, j)$, in the $\xi$-and $\zeta$-directions, respectively, and denote the solution on this grid by $h(i)$ and $\Theta(i, j)$. Vertical thermal diffusion is handled by second-order-accurate finite differences on a grid determined by Chebyshev collocation. We use a Petrov-Galerkin scheme to handle radial derivatives in the $h$ equation, and a Van Leer flux-limiting algorithm for the advective terms in the heat equation; neither of these discretizations requires the addition of an explicit linear diffusion to operate efficiently. The entire computational algorithm is coded in Matlab and makes use of that software's existing routines. We tested the accuracy of the various discretizations against several model problems and special limits, and checked the numerical integration as a whole using global integrals of the equations (namely mass and energy conservation). The computational scheme is summarized as follows:

(a) Equation of motion of the fluid edge. At the contact radius $r=R(t)$, $h(\xi=1, t)=h_{w}$. We differentiate in time: $h_{t}(\xi=1, t)=0$. From equation (A 1$)$, the contact radius therefore evolves as

$$
R_{t}=\left[\frac{1}{h_{\xi}} \partial_{\xi}(\xi \psi)\right]_{\xi=\zeta=1}=\left[u-\frac{R w}{h_{\xi}}\right]_{\xi=\zeta=1},
$$

provided the source $\Omega$ vanishes at the edge. We time step this equation along with the discretized forms of the partial differential equations.

(b) Vertical discretization and evaluation of integrals in (A 3)-(A 4). The function $\Upsilon_{\zeta}$ is approximated by Chebyshev polynomials and the integrals in (A 3)-(A 4) are numerically evaluated (Press et al. 1992). For this purpose, the computational points are collocated along the $\zeta$-axis at the roots of Chebyshev polynomials. We also include the points, $\zeta=0$ and 1 , in order to impose directly the boundary conditions. The total number of grid points in $\zeta$ is $N_{\zeta}$.

(c) Vertical diffusion in (A 2). The Chebyshev collocation is also used in the discretization of the vertical diffusive term in the heat equation, which is dealt with using the finite-difference second-order three-points formula,

$$
\Theta_{\zeta \zeta}(i, j)=\frac{2 \Theta(i, j-1)}{\left(\zeta_{j+1}-\zeta_{j-1}\right)\left(\zeta_{j}-\zeta_{j-1}\right)}-\frac{2 \Theta(i, j)}{\left(\zeta_{j+1}-\zeta_{j}\right)\left(\zeta_{j}-\zeta_{j-1}\right)}+\frac{2 \Theta(i, j+1)}{\left(\zeta_{j+1}-\zeta_{j-1}\right)\left(\zeta_{j+1}-\zeta_{j}\right)} .
$$

Typically, discretizations with as few as 20 points were sufficient to compute both the integrals for the velocity field and the vertical diffusion.

(d) Collocation in radius. The points along the $\xi$-axis are collocated in $[0,1)$ according to the relation $\mathrm{d} \xi_{n} / \mathrm{d} n=\varsigma(n-1)^{N_{0}}\left(n-N_{\xi}\right)^{N_{1}}$, where $n$ is the point index and $\varsigma$ is set so that $\xi_{N_{\xi}}=1$. By varying the exponents $N_{0}$ and $N_{1}$ we may increase the densities of points near the ends of the domain. Both are critical for resolution: at the fluid edge $\xi \approx 1, h_{\xi}$ needs to be resolved, while we must sample the vent region properly in order to keep the mass flux constant. As a rule of thumb, we found that $N_{\xi}=80$ to 120 points were sufficient. We also recommend $N_{0}=1$ and $N_{1}=1$ for $B \approx 0, N_{1}=2$ for $B \approx 10$, and $N_{1}=3$ for $B \approx 100$. 
(e) Mass flux terms in (A 1). The spatial discretization of the $h$-equation is performed using a second-order Petrov-Galerkin method suggested by Skeel \& Berzins (1990); the scheme automatically incorporates the coordinate singularity at the origin. First, two sets of quadrature points are generated:

$$
\hat{\xi}_{i+1 / 2}=\frac{2}{3} \frac{\xi_{i+1}^{2}+\xi_{i+1} \xi_{i}+\xi_{i}^{2}}{\xi_{i+1}+\xi_{i}}, \quad \check{\xi}_{i+1 / 2}^{2}=\frac{1}{2} \frac{\xi_{i+1}^{2}-\xi_{i}^{2}}{\log \xi_{i+1}-\log \xi_{i}},
$$

with $\check{\xi}_{3 / 2}=0$. Then, $h$ and $h_{\xi}$ are evaluated at the first of these quadrature points by the interpolation formulae,

$h\left(\hat{\xi}_{i+1 / 2}\right)=h(i)+\frac{\hat{\xi}_{i+1 / 2}^{2}-\xi_{i}^{2}}{\xi_{i+1}^{2}-\xi_{i}^{2}}[h(i+1)-h(i)], \quad h_{\xi}\left(\hat{\xi}_{i+1 / 2}\right)=\frac{2 \hat{\xi}_{i+1 / 2}}{\xi_{i+1}^{2}-\xi_{i}^{2}}[h(i+1)-h(i)]$.

We use a similar construction to evaluate the integral, $\Upsilon$, at the quadrature points, but this further requires $\eta$ and therefore $h_{\xi}$ : Guided by (A 8), we use the average,

$$
h_{\xi}(i)=\frac{\xi_{i}}{\xi_{i+1}+\xi_{i}} \frac{h(i+1)-h(i)}{\xi_{i+1}-\xi_{i}}+\frac{\xi_{i}}{\xi_{i}+\xi_{i-1}} \frac{h(i)-h(i-1)}{\xi_{i}-\xi_{i-1}} .
$$

We next compute the flux and source terms in (A 1) at $\hat{\xi}_{i+1 / 2}$, denoted $g_{i+1 / 2}$ and $f_{i+1 / 2}$, respectively. Thence,

$$
\begin{aligned}
& h_{t}(i)= \frac{2}{\check{\xi}_{i+1 / 2}^{2}-\check{\xi}_{i-1 / 2}^{2}}\left(\frac{g_{i+1 / 2} \check{\xi}_{i+1 / 2}^{2}}{\hat{\xi}_{i+1 / 2}}-\frac{g_{i-1 / 2} \check{\xi}_{i-1 / 2}^{2}}{\hat{\xi}_{i-1 / 2}}\right)+\frac{\check{\xi}_{i+1 / 2}^{2}-\xi_{i}^{2}}{\check{\xi}_{i+1 / 2}^{2}-\check{\xi}_{i-1 / 2}^{2}} f_{i+1 / 2} \\
&+\frac{\xi_{i}^{2}-\check{\xi}_{i-1 / 2}^{2}}{\check{\xi}_{i+1 / 2}^{2}-\check{\xi}_{i-1 / 2}^{2}} f_{i-1 / 2}, \\
& h_{t}(1)=2 \frac{g_{3 / 2}}{\hat{\xi}_{3 / 2}}+f_{3 / 2} .
\end{aligned}
$$

An analogous procedure allows us to discretize the equations for $R_{t}$ :

$$
R_{t}=-R\left[\frac{2 g_{N_{\xi}+1 / 2} \check{\xi}_{N_{\xi}+1 / 2}^{2} \hat{\xi}_{N_{\xi}+1 / 2}^{-1}-2 g_{N_{\xi}-1 / 2} \check{\xi}_{N_{\xi}-1 / 2}^{2} \hat{\xi}_{N_{\xi}-1 / 2}^{-1}}{\left(\check{\xi}_{N_{\xi}+1 / 2}^{2}-1\right) h_{\xi}\left(\hat{\xi}_{N_{\xi}+1 / 2}\right) \hat{\xi}_{N_{\xi}+1 / 2}+\left(1-\check{\xi}_{N_{\xi}-1 / 2}^{2}\right) h_{\xi}\left(\hat{\xi}_{N_{\xi}-1 / 2}\right) \hat{\xi}_{N_{\xi}-1 / 2}}\right]
$$

( $f$ ) Discretization of the radial advection terms in (A 2). The discretization of the advection terms in the heat equation must cope with the sharp temperature gradients that are advected with the flow. A simple first-order advective scheme would introduce a large numerical diffusion that may well become bigger than the physical one. On the other hand, any linear second-order scheme has problems in dealing with large gradients and would inevitably produce spurious oscillations at the discontinuities. We therefore opted to use a Van Leer flux-limiter (Hirsch 1988; LeVeque 1992), which is second-order accurate and 'total variation diminishing', even in non-conservative form (Zvan, Forsyth \& Vetzal 1998). The derivative, $\Theta_{\xi}$, is approximated with the second-order centred formula,

$$
\Theta_{\xi}(i, j)=\frac{\Theta\left(i+\frac{1}{2}, j\right)-\Theta\left(i-\frac{1}{2}, j\right)}{\xi_{i+1 / 2}-\xi_{i-1 / 2}},
$$


where $\xi_{i+1 / 2}=\left(\xi_{i+1}-\xi_{i}\right) / 2$. The value of the temperature at the mid-points depends on the sign of the velocity $\hat{u}=\left(u-\xi R_{r}\right) / R$, in an upwind fashion: If $\hat{u}_{i, j} \geqslant 0$

$$
\begin{gathered}
\Theta\left(i+\frac{1}{2}, j\right)=\Theta(i, j)+\phi\left(q_{i+1 / 2, j}\right) \frac{\Theta(i+1, j)-\Theta(i, j)}{2}, \\
q_{i+1 / 2, j}=\frac{\Theta(i, j)-\Theta(i-1, j)}{\xi_{i}-\xi_{i-1}} \frac{\xi_{i+1}-\xi_{i}}{\Theta(i+1, j)-\Theta(i, j)},
\end{gathered}
$$

and if $\hat{u}_{i, j}<0$,

$$
\begin{gathered}
\Theta\left(i+\frac{1}{2}, j\right)=\Theta(i+1, j)-\phi\left(q_{i+1 / 2, j}\right) \frac{\Theta(i+1, j)-\Theta(i, j)}{2} \\
q_{i+1 / 2, j}=\frac{\Theta(i+2, j)-\Theta(i+1, j)}{\xi_{i+2}-\xi_{i+1}} \frac{\xi_{i+1}-\xi_{i}}{\Theta(i+1, j)-\Theta(i, j)} .
\end{gathered}
$$

The function $\phi=(|q|+q) /(1+|q|)$ is the 'Van Leer flux limiter'. The scheme is applied in non-conservative form to avoid numerical cancellations.

A similar approximation was used for the $\zeta$ advective term, but the computation of the corresponding velocity,

$$
\hat{w}=\frac{1}{h}\left\{\frac{1}{\xi} \partial_{\xi}\left[\xi \frac{\zeta \psi(\xi, 1)-\psi}{R}\right]+(1-\zeta) \Omega\right\} \equiv \frac{1}{h}\left(\frac{1}{\xi} \partial_{\xi} \mathscr{G}+\mathscr{F}\right),
$$

deserves further mention. To be consistent with the treatment of the $h$-equation, at each vertical collocation point a Petrov-Galerkin-like discretization was employed:

$$
\begin{gathered}
\hat{w}_{i, j} \frac{2 \check{\xi}_{i+1 / 2}^{2} \mathscr{G}_{i+1 / 2, j} / \hat{\xi}_{i+1 / 2}-2 \check{\xi}_{i-1 / 2}^{2} \mathscr{G}_{i-1 / 2, j} / \hat{\xi}_{i-1 / 2}+\left(\check{\xi}_{i+1 / 2}^{2}-\xi_{i}^{2}\right) \mathscr{F}_{i+1 / 2, j}+\left(\xi_{i}^{2}-\check{\xi}_{i-1 / 2}^{2}\right) \mathscr{F}_{i-1 / 2, j}}{h(i)\left(\check{\xi}_{i+1 / 2}^{2}-\check{\xi}_{i-1 / 2}^{2}\right)} \\
\hat{w}_{1, j}=\frac{1}{h(1)}\left(2 \frac{\mathscr{G}_{3 / 2, j}}{\hat{\xi}_{3 / 2}}+\mathscr{F}_{3 / 2, j}\right)
\end{gathered}
$$

( $g$ ) Excessive edge gradients. Outside the dome, $h$ is by definition zero. However, this introduces difficulties because the height gradient can then become singular at the edge of the dome (see Balmforth et al. 2000). To avoid this problem, we 'pre-wet' the surface with a thin film of fluid, and introduce a certain number of 'ghost points', located inside the film (usually 3). At these ghost points, we take $\xi \geqslant 1, h=h_{w}, \eta=1$ and

$$
\Theta(\zeta)=\Theta_{b}+\zeta^{2}\left(\Theta_{s}-\Theta_{b}\right), \quad \Theta_{b}=\min \left(\Theta_{s}+\frac{h_{w}}{2} \Theta_{s} ; 1\right), \quad \Theta_{s}=\exp \left(-\sqrt{\kappa} t / h_{w}\right)
$$

which is based on an integral-balance solution of the heat equation (computations with the full diffusion equation confirm its accuracy). We typically use $h_{w}=10^{-4}$, but different choices make negligible difference to the outcome.

(h) Thermal boundary conditions. The thermal boundary conditions are:

$$
\begin{gathered}
\Theta_{\xi}(1, j)=\Theta_{\xi}\left(N_{\xi}+2, j\right)=0, \quad \Theta_{\zeta}\left(i, N_{\zeta}\right)=-[h(i) / \sqrt{\kappa}] \Theta\left(i, N_{\zeta}\right), \\
\Theta(i, 1)=1 \quad \text { for } \xi_{i} \leqslant r_{*} / R, \quad \Theta_{\zeta}(i, 1)=0 \text { for } \xi_{i}>r_{*} / R .
\end{gathered}
$$


These conditions are directly applied when constructing the advective terms. For the vertical diffusion term:

$$
\begin{gathered}
\Theta_{\zeta \zeta}(i, 1)=2 \frac{\Theta(i, 2)-\Theta(i, 1)}{\zeta_{2}^{2}} \quad \text { for } \xi_{i}>r_{*} / R \\
\Theta_{\zeta \zeta}\left(i, N_{\zeta}\right)=-2 \frac{\Theta\left(i, N_{\zeta}\right)-\Theta\left(i, N_{\zeta}-1\right)}{\left(\zeta_{N_{\zeta}}-\zeta_{N_{\zeta}-1}\right)^{2}}-\frac{2 h(i)}{\sqrt{\kappa}} \frac{\Theta\left(i, N_{\zeta}\right)}{\left(\zeta_{N_{\zeta}}-\zeta_{N_{\zeta}-1}\right)},
\end{gathered}
$$

which are second-order approximations, consistent with (A 6). Over the vent region, $\Theta$ is specified, so we need no analogue of the first of these relations.

(i) Initial conditions. At $t=0$, the dome starts expanding from $R=r_{*}$ with $h=h_{w}$ and $\Theta=1$.

( $j$ ) Time integrator. The discretized system of equations is integrated numerically using fifth-order backward differentiation formulae (Gear's method). The sparsity pattern of the Jacobian matrix generated from the right-hand sides of the equations is exploited explicitly to reduce numerical computation and memory requirements. Also the variables are reordered to speed up the LU factorization of the sparse system.

\section{A.1. Schemes for the skin models}

The two codes used for the skin models are as follows: The first code uses the adaptive scheme of Blom \& Zegeling (1994). To ensure the efficient performance of the adaptive code, we pre-wet the plane with a thin film of fluid $\left(h(r, 0)=2 \times 10^{-4}\right)$ and add linear diffusion terms to both the $h$ and $\mathscr{E}$ equation (with diffusivities $\left.3 \times 10^{-4} /[1+10 \max (t, 1)]\right)$. We impose the boundary conditions, $h_{r}=\Theta_{r}=u_{b}=0$ at $r=0$, and $h=2 \times 10^{-4}$ and $\Theta_{s}=u_{b}=0$ at the edge of the computational domain. (For the skin model without extensional stresses, $u_{b}$ is prescribed and needs no boundary conditions.) We use an expanding coordinate system in which, at each timestep, the domain expands at a rate given by the speed of the dome's edge (defined as the radius where $h=0.1)$ at the previous timestep. We begin the computation with a domain size of radius $1.1 r_{*}$.

The second scheme is a Petrov-Galerkin scheme for spatial discretization coupled with a Gear solver in time, along the lines described earlier. The Petrov-Galerkin scheme does not require additional diffusion or pre-wetting, and at the edge we impose $h=\Theta_{s}=u_{b}=0$. At $r=0$, the boundary conditions are the same as in the other scheme. The computational domain is again expanding; we solve explicitly an equation of motion for edge, as discussed earlier. Both simulations are orders of magnitude faster than those for the full shallow-lava model.

\section{A.2. Temperature-dependent yield stress}

When the yield stress depends on the temperature, we define

$$
\tau_{p}(T)=\tau_{p}\left(T_{e}\right) g(\Theta), \quad B=\frac{H \tau_{p}\left(T_{e}\right)}{\eta_{e} U},
$$

(cf. (2.5)) with $g(1)=1$, to isolate the temperature variation of the yield stress from its characteristic value at the eruption temperature. For illustration, we take $g(\Theta)=\exp \left(\gamma_{B}(1-\Theta)\right)$, with $\gamma_{B}$ a parameter. The (fake) yield condition, $\tau \geqslant \tau_{p}$, now becomes

$$
\tau=\left|\tau_{r z}\right|=\left|h_{r}\right|(h-z) \geqslant B g(\Theta) .
$$


Because $\Theta$ varies with depth, this condition is no longer straighforward to implement, and the location of a fake yield surface must follow from solving the implicit equation,

$$
\left|h_{r}\right|(h-Y)=B g[\Theta(r, Y, t)] .
$$

In fact, in principle, there may be multiple solutions to this equation (which we label by $z=Y_{l}$, for $\left.l=1,2, \ldots\right)$, revealing the presence of more than one pseudo-plug. We find numerically that such regions do indeed appear (see $\S 6$ ).

The yield condition can be written in the form,

$$
\mathscr{C}=\mathscr{Y}(z)-z \equiv h-\frac{B g(\Theta)}{\left|h_{r}\right|}-z \geqslant 0,
$$

from which the velocity field follows from

$$
u_{z}=-h_{r} \begin{cases}0 & \text { for } \mathscr{C}<0, \\ {[\mathscr{Y}(z)-z] / \mathscr{A}(\Theta)} & \text { for } \mathscr{C} \geqslant 0 .\end{cases}
$$

As regards numerical implementation, the fake yield surfaces are found by Newton iteration using Chebyshev interpolation between the grid points in $\zeta=z / h$. Note that, because the grid points at $\zeta=0$ and 1 are not Chebyshev collocation points, the Chebyshev interpolation can fail in their vicinity; to avoid this problem, we use a linear interpolant for $\zeta_{1}=0<\zeta<\zeta_{2}$ and $\zeta_{N_{\zeta}-1}<\zeta<\zeta_{N_{\zeta}}=1$. To improve further the accuracy, we evaluate the integrals over $\zeta$ by first splitting them at all the fake yield surfaces.

\section{REFERENCES}

Balmforth, N. J., Burbidge, A. S. \& Craster, R. V. 2001 Shallow lava theory. In Geomorphological Fluid Mechanics (ed. N. J. Balmforth \& A. Provenzale), pp. 164-187. Springer.

Balmforth, N. J., Burbidge, A. S., Craster, R. V., Salzig, J. \& Shen, A. 2000 Visco-plastic models of isothermal lava domes. J. Fluid Mech. 403, 37-65.

BAlmforth, N. J. \& Craster, R. V. 1999 A consistent thin-layer theory for Bingham fluids. J. Non-Newtonian Fluid Mech. 84, 65-81.

Balmforth, N. J. \& Craster, R. V. 2000 Dynamics of cooling domes of viscoplastic fluid. J. Fluid Mech. 422, 225-248, referred to here in as paper 1.

Bercovici, D. \& Lin, J. 1996 A gravity current model of cooling mantle plume heads with temperature-dependent buoyancy and viscosity. J. Geophys. Res. Solid Earth 101, 3291-3309.

BlaKe, S. 1990 Viscoplastic models of lava domes. In Lava Flows and Domes: Emplacement Mechanisms and Hazard Implications (ed. J. H. Fink), pp. 88-128. IAVCEI Proc. in Volcanology, vol. 2, Springer.

Blom, J. G. \& Zegeling, P. A. 1994 Algorithm 731: a moving-grid interface for systems of onedimensional time-dependent partial differential equations. ACM Trans. Math. Software 20, 194-214.

Braun, R. J., Murray, B. T., Boettinger, W. J. \& McFadden, G. B. 1995 Lubrication theory for reactive spreading of a thin drop. Phys. Fluids 7, 1797-1810.

Chomaz, J. M. 2001 The dynamics of a viscous soap film with soluble surfactant. J. Fluid Mech. 442, 387-409.

Dinh, T. N., Konovalikhin, M. J. \& Sehgal, B. R. 2000 Core melt spreading on a reactor containment floor. Prog. Nuclear Engng 36, 405-468.

Ehrhard, P. \& Davis, S. H. 1991 Non-isothermal spreading of liquid drops on horizontal plates. J. Fluid Mech. 220, 365-388.

Erneux, B. T. \& Davis, S. H. 1993 Nonlinear rupture of free films. Phys. Fluids A 5, 1117-1122.

Griffiths, R. W. 2000 The dynamics of lava flows. Annu. Rev. Fluid Mech. 32, 477-518.

Griffiths, R. W. \& Fink, J. H. 1993 Effects of surface cooling on the spreading of lava flows and domes. J. Fluid Mech. 252, 667-702. 
Griffiths, R. W. \& Fink, J. H. 1997 Solidifying Bingham extrusions: a model for the growth of silicic lava domes. J. Fluid Mech. 347, 13-36.

Hirsch, C. 1988 Numerical Computation of Internal and External Flow. Vol 2. Computational Methods for Inviscid and Viscous Flows. Wiley.

Hosol, A. E. \& Bush, J. W. M. 2001 Evaporative instabilities in climbing films. J. Fluid Mech. 442, 217-239.

HuPPERT, H. E. 1982 The propagation of two-dimensional and axisymmetric viscous gravity currents over a rigid horizontal surface. J. Fluid Mech. 121, 43-58.

HuTter, K. 1983 Theoretical Glaciology. D. Reidel.

Jensen, O. E. \& GrotberG, J. B. 1993 The spreading of heat or soluble surfactant along a thin film. Phys. Fluids A 5, 58-68.

King, J. R., Riley, D. S. \& Sansom, A. 2000 Gravity currents with temperature-dependant viscosity. Comput. Assist. Mech. Engng Sci. 7, 251-277.

LeVeque, R. J. 1992 Numerical Methods for Conservation Laws. Birkhäuser.

Lister, J. R. \& KeRR, R. C. 1994 Influence of cooling on lava flow dynamics - comment. Geology 22, 93-94.

NerI, A. 1998 A local heat transfer analysis of lava cooling in the atmosphere: application to thermal diffusion-dominated lava flows. J. Volcan. Geotherm. Res. 81, 215-243.

Oron, A., Davis, S. H. \& Bamkoff, S. G. 1997 Long-scale evolution of thin liquid films. Rev. Mod. Phys. 69, 931-980.

Oron, A. \& Rosenau, P. 1994 On a nonlinear thermocapillary effect in thin liquid layers. J. Fluid Mech. 273, 361-374.

ÖZışıK, M. N. 1968 Boundary Value Problems of Heat Conduction. Dover.

Papageorgiou, D. T. 1995 On the breakup of viscous liquid threads. Phys. Fluids 7, 1529-1544.

Press, W. H., Teukolsky, S. T., Vetterling, W. T. \& Flannery, B. P. 1992 Numerical Recipes in $C$ : the Art of Scientific Computing. Cambridge University Press.

Reisfeld, B., Bankoff, S. G. \& Davis, S. H. 1991 The dynamics and stability of thin liquid films during spin coating. I films with constant rates of evaporation or adsorption. J. Appl. Phys. 70, 5258-5277.

Skeel, R. D. \& Berzins, M. 1990 A method for the spatial discretization of parabolic equations in one space variable. SIAM J. Sci. Stat. Comput. 11, 1-32.

Stasiuk, M. V., Jaupart, C. \& Sparks, R. S. J. 1993 Influence of cooling on lava-flow dynamics. Geology 21, 335-338.

Zvan, R., Forsyth, P. A. \& Vetzal, K. 1998 Robust numerical methods for PDE models of Asian options. J. Comput. Finance 1, 39-78. 